\title{
Fuzzy Reasoning Method based on Distance Measure and its Reductive Property
}

\author{
SONIL KWAK ${ }^{1 *}$, UNHA KIM $^{1}$, KUMJU KIM $^{1}$, ILMYONG SON $^{1}$, CHONGHAN RI $^{1}$ \\ ${ }^{1}$ Faculty of Information Science \\ Kim Il Sung University, Pyongyang, DPR KOREA
}

\begin{abstract}
This paper shows a basic and original fuzzy reasoning method that can draw a novel study direction of the approximate inference in fuzzy systems with uncertainty. Firstly we propose a criterion function for checking of the reductive property about fuzzy modus ponens (FMP) and fuzzy modus tollens (FMT). Secondly unlike fuzzy reasoning methods based on the similarity measure, we propose a principle of new fuzzy reasoning method based on distance measure and then present two theorems for FMP and FMT. Thirdly through the several computational experiments, we show that proposed method is simple and effective, and in accordance with human thinking. Finally we pointed out conclusion that proposed method does satisfy the convergence of the fuzzy control and has not information loss.
\end{abstract}

Key-Words: - Fuzzy Reasoning, Normal Fuzzy Set, Distance Measure, Fuzzy Modus Ponens, Fuzzy Modus Tollens, Reductive Property

Received: March 31, 2019. Revised: July 1, 2020. Accepted: July 7, 2020. Published: July 15, 2020.

\section{Introduction}

Fuzzy modus ponens (FMP) and fuzzy modus tollens (FMT) are two fundamental patterns of general fuzzy reasoning [32]. In [32] Lotfi A. Zadeh proposed the Compositional Rule of Inference (CRI) for FMP and FMT. In [26] Wang presented the Triple Implication Principle (TIP) with total inference rules of fuzzy reasoning. Since the inception of the triple I method [26], many papers have researched the fuzzy inference method $[1,3,4,5,7,8,10,15,19,23,34]$. However unfortunately Lotfi (Zadeh) was not discovered that that the underlying semantic of his's CRI is unclear, ourselves authors find that Lotfi (Zadeh)'s fuzzy reasoning result does completely not satisfy the reductive property.

Reductive Property is one of the essential and important properties in the applications of the fuzzy inference mechanism [2,6,9,11-14,18,21,24-27,30].

This paper shows a basic and original fuzzy reasoning method that can draw a novel study direction of the approximate inference in fuzzy systems with uncertainty. For realization of this syudy direction this work is based on the recent paper's idea presented by the several authors, which is to obtain a new conclusion by the vertical moving distance operation between the antecedent and the given premise(observation), which is based on the paper [10]; "Son-Il Kwak, Un-Sok RYU, Kum-Ju KIM, and Myong-Hye JO, A Fuzzy reasoning Method based on Compensating Operation and its
Application to Fuzzy Systems, Iranian Journal of Fuzzy Systems, 16(3), pp. 17-34, 2019”. This paper is based on [10].

In this paper we propose a new criterion function for checking of the reductive property about the fuzzy reasoning result for fuzzy modus ponens and fuzzy modus tollens. And then, unlike fuzzy reasoning methods based on the similarity measure, we propose a new fuzzy reasoning method based on distance measure (DM) presented in [21].

The rest of this paper is organized as follows. In section 2, we discuss backgrounds for FMP and FMT about the fuzzy reasoning methods based on fuzzy relation. In section 3, a new criterion function and fuzzy reasoning method are presented, respectively. In section 4 , the reductive property of CRI and TIP, AARS and our method are checked.

\section{Backgrounds}

Generally known fuzzy reasoning methods are FMP and FMT in the fuzzy system with 1 input 1 output 1 rule. General form of FMP in [5] is as follows.

Rule; if $x$ is $A$ then $y$ is $B$, Premise: $x$ is $A^{*}$,

Conclusion: $y$ is $B^{*}$

General form of FMT in the paper [5] is as follows.

$$
\begin{aligned}
& \text { Rule; if } x \text { is } A \text { then } y \text { is } B \text {, Premise: } y \text { is } B^{*} \text {, } \\
& \text { Conclusion: } x \text { is } A^{*}
\end{aligned}
$$

, where $A^{*} \in F(X), A \in F(X)$ are fuzzy sets defined in the universe of discourse $X, B^{*} \in F(Y), B \in F(Y)$ are fuzzy sets defined in the universe of discourse $Y$. In the fuzzy system with 1 input loutput $\mathrm{n}$ rules, 
we rewrite the definition for reductive property of fuzzy inference method in [5]. According to [5,10], the equation (2) can be written as follows, because FMT is opposite to FMP.

$$
\begin{aligned}
& \text { Rule; if } y \text { is } \bar{B} \text { then } x \text { is } \bar{A} \text {, Premise: } y \text { is } \bar{B} \text {, } \\
& \text { Conclusion: } x \text { is } \bar{A}
\end{aligned}
$$

where $\bar{A}=1-A, \bar{B}=1-B$. For equation (1), (2), an $\mathrm{d}$ (3), according to Zadeh's viewpoint, Rule is $\mathrm{r}$ epresented by some fuzzy relation. For example, where $\rightarrow$, is Zadeh's implication, the fuzzy rel ation of the rule is presented as follows. $R(x, y)=A(x) \rightarrow_{z} B(y), \quad a \rightarrow_{z} b=(1-a) \vee(a \wedge b) \quad(4)$

In the paper [34], authors listed 4 most important implication operators and the corresponding t-norms. As mentioned in [34], Łukasiewicz's implication $a \rightarrow_{L} b$ and the corresponding t-norm $a \otimes_{L} b$, Gödel's $a \rightarrow_{G} b$ and $a \otimes_{G} b$, R0's $a \rightarrow_{R_{0}} b$ and $a \otimes_{R_{0}} b$, and Gougen's $a \rightarrow_{G_{0}} b$ and $a \otimes_{G_{0}} b$ are described as follows, respectively.

$$
\begin{aligned}
& a \rightarrow_{L} b=1 \wedge(1-a+b), \quad a \otimes_{L} b=0 \vee(a+b-1) \\
& a \rightarrow_{G} b=\left\{\begin{array}{lll}
1, & \text { if } & a \leq b \\
b, & \text { if } & a>b
\end{array}, \quad a \otimes_{G} b=a \wedge b\right. \\
& a \rightarrow_{R_{0}} b=\left\{\begin{array}{ccc}
1, & \text { if } & a \leq b \\
a^{\prime} \vee b, & \text { if } & a>b
\end{array}, \quad a \otimes_{R_{0}} b=\left\{\begin{array}{ccc}
0, & \text { if } & a+b \leq 1 \\
a \wedge b, & \text { if } & a+b>1
\end{array}\right. \text {, }\right. \\
& a^{\prime}=1-a \\
& a \rightarrow_{G_{0}} b=\left\{\begin{array}{lll}
1, & \text { if } & a \leq b \\
\frac{b}{a}, & \text { if } & a>b
\end{array}, \quad a \otimes_{G_{0}} b=a b\right.
\end{aligned}
$$

\section{New Reductive Property Criterion Function and Fuzzy Reasoning Method}

\subsection{Motivation and Importance of New Fuzzy Reasoning Method}

\subsubsection{Motivation}

The reductive property is one of the essential and important properties in the fuzzy reasoning $[18,30]$. But a lot of fuzzy reasoning methods have some shortcomings. The motivations obtained from some shortcomings are as follows.

- As mentioned in [21,22], the underlying semantic of CRI is unclear, its reasoning result does completely not satisfy the reductive property. Therefore reasoning method that does satisfy the reductive property must be studied.

- As pointed out in [8], shortcoming of TIP is that it cannot be applied in fuzzy control. Therefore fuzzy reasoning method that can be applied in fuzzy control must be researched.

- As presented in [17], the some fuzzy reasoning methods based on the fuzzy relation have the contradict that they can be applied to the practical problem, for example fuzzy control, but do not satisfy the reductive property, vice versa. Therefore fuzzy reasoning method that has not some contradict must be studied.

- As mentioned in [23], the fuzzy reasoning methods based on similarity measure (SM) depend strongly on the similarity measure and the modification function, and do not completely satisfy the reductive property. Therefore fuzzy reasoning method that does not depend on the similarity measure and the modification function must be researched.

- As presented in [26], due to many fuzzy reasoning methods based on SM do use nonlinear operators, the fuzzy sets of reasoning result are non-normal and non-convex ones. Therefore in fuzzy reasoning processing, linear operators must possibly be used.

- According to [2,6,13-16], a lot of fuzzy reasoning methods mathematically seem that they are all accompanied with a common shortcoming, that is, information loss. One of the reasons that do not satisfy the reductive property is to refer to losses of information occurred in reasoning processes. Therefore, information loss must possibly be reduced in fuzzy reasoning processing.

- As shown in $[5,14,15,18]$, the criterion function for checking of fuzzy reasoning results has only 2 values, i.e., ' 1 ' or ' $O$ ' for satisfaction of the reductive property, ' 0 ' or ' $x$ ' for non-satisfaction of one. That is, this evaluation is too strict for the reductive property. Therefore criterion function for checking of fuzzy reasoning result must possibly be defined flexibly.

Comprehensively, in order to overcome existing shortcomings presented in $[2,5,6,8,13-17,18,21$, 22,24 , and 30], fuzzy reasoning method based on new methodology or idea must be developed without some losses of information and with smooth evaluation for the reductive property. From the above mentioned facts, we try to develop a new fuzzy reasoning method with respect to the information loss and reductive property. This is a motivation of this paper. 


\subsubsection{Possibility and Importance}

In a lot of papers fuzzy reasoning methods based on SM are proposed. Their basic idea is to consider the similarity measure of the consequent $B(y)$ and the fuzzy reasoning conclusion $B^{*}(y)$ if the antecedent $A(x)$ is similar to the given premise $A^{*}(x)$ for FMP. This idea is right. By the way we consider following:

- Similarity measure and distance measure have inverse proportional relation. That is, "the antecedent $A(x)$ is similar to the given premise $A^{*}(x)$ " is approximately equal to "the antecedent $A(x)$ is closer to the given premise $A^{*}(x)$ ". Here "similar" is correspondent to similarity measure, "closer" to distance measure. if $A(x)$ is completely equal to $A^{*}(x)$ then the similarity measure is 1 and distance measure is 0 .

- The fuzzy reasoning methods based on similarity do not require the calculation of fuzzy relation or implication. However the fuzzy reasoning results obtained by the similarity methods depend strongly on the similarity measure and the modification function.

- The fuzzy reasoning methods based on similarity do use nonlinear, i.e., max, min operator. Thus fuzzy reasoning methods based on similarity measure have a lot of information loss [26]. But fuzzy reasoning methods based on distance measure can be used linear operator for example summation and subtraction, thereby information loss can be reduced.

- And the similarity measure has closed interval $[0,1]$ and distance measure $[0, \mathrm{~m}]$, where $m$ is a finite number, $m>0$.

According to these facts, fuzzy reasoning based on distance measure (DM) is possible. This is a possibility and an importance of our paper.

\subsection{Reductive Property Criterion Function}

The reductive property is one of the essential properties in the applications of the fuzzy inference mechanism [5,16]. According to [16,28], four cases of the premise for FMP in Class 1 are as follows;

Case 1: $A^{*}$ is $A$,

Case 2: $A^{*}$ is very $A\left(=A^{2}\right)$,

Case 3: $A^{*}$ is more or less $A\left(=A^{1 / 2}\right)$,

Case 4: $A^{*}$ is not $A(=1-A)$.

Since FMT is opposite to FMP, according to
$[16,28]$, four cases of the given premise for FMT in Class 1 are as follows;

Case 6: $B^{*}$ is not $B(=1-B)$,

Case 7: $B^{*}$ is not very $B\left(=1-B^{1 / 2}\right)$,

Case 8: $B^{*}$ is not more or less $B\left(=1-B^{1 / 2}\right)$,

Case 9: $B^{*}$ is $B$.

And four cases of the Premise for FMP in Class 2 are as follows;

Case 1: $A^{*}$ is $A$,

Case 2: $A^{*}$ is very $A\left(=A^{2}\right)$,

Case 3: $A^{*}$ is more or less $A\left(=A^{1 / 2}\right)$,

Case 5: $A^{*}=$ slightly tilted of $A(=$ s.t. $A)$.

And four cases of the given premise for FMT in Class 2 are as follows;

Case 6: $B^{*}$ is not $B(=1-B)$,

Case 7: $B^{*}$ is not very $B\left(=1-B^{2}\right)$,

Case 8: $B^{*}$ is not more or less $B\left(=1-B^{1 / 2}\right)$,

Case 10: $B^{*}=$ slightly tilted of $B(=$ s.t. $B)$.

What conclusion $B^{*}$ for FMP and $A^{*}$ for FMT can be obtain? For this, Table 1 shows reductive property of FMP and FMT based on [5, 18]. In Table 1, Case 4 and 8 are criterion based on the paper [18], Case 5 and 10 criterion based on the paper [5], for FMP and FMT, respectively. In other words, Case 1, Case 2, Case 3, and Case 4 are criterion functions based on the paper [18], Case 6, Case 7, Case 8, and Case 10 are criterion functions based on the paper [5]. In the paper [5], authors mentioned that their proposed method is based on the assumption that the premise $A^{*}$ is slightly different from the antecedent of fuzzy rule $A$ and thus the conclusion $B^{*}$ is slightly different from the consequent $B$ of fuzzy rule, therefore, they do not expect a reasonable conclusion if the premise $A^{*}$ is different from the antecedent $A$ too much. Unlike the classical reasoning, if the given premise $A^{*}$ is not exactly equal to the antecedent $A$, we can still obtain fuzzy reasoning result $B^{*}$. However we know that if the given premise $A^{*}$ and the antecedent $A$ are totally different, then the fuzzy reasoning result $B^{*}$ might be unreasonable or uninformative. Then in practical applications, a group of fuzzy rules called rule base is used to avoid the incorrect fuzzy reasoning result caused by the deviation between the given premise $A^{*}$ and the antecedent $A$. As obviously mentioned in the paper [9], if the given premise $A^{*}$ is slightly different from the antecedent $A$ then the fuzzy reasoning conclusion $B^{*}$ is slightly different from the consequent $B$. According to combination of the paper [5] and [18], for example the antecedent fuzzy 
$A=[$ small $]$ and consequent fuzzy set $B=[$ large $], \quad$ we can obtain the following Table 1.

Table 1 New Reductive property criterion for FMP and FMT based on $[5,18]$

\begin{tabular}{l|c|c|l}
\hline \multirow{2}{*}{ FMP } & \multicolumn{3}{|c}{ if $x$ is $A$ then $y$ is $B$} \\
\cline { 2 - 5 } & $x$ is $A^{*}[5,18]$ & $y$ is $B^{*}[5,18]$ & New Reductive property criterion $R P C F_{F M P}$ of $y$ is $B^{*},(\%)$ \\
\hline Case 1 & $A^{*}=A$ & $B^{*}=B$ & $\left(1-\sum_{k=1}^{r}\left|b_{k l}^{*}-b_{k}\right| / r\right) \times 100$ \\
\hline Case 2 & $A^{*}=A^{2}$ & $B^{*}=B^{2}$ or $B$ & $\left(1-\sum_{k=1}^{r}\left|b_{k l}^{*}-b_{k}^{2}\right| / r\right) \times 100$ or $\left(1-\sum_{k=1}^{r}\left|b_{k l}^{*}-b_{k}\right| / r\right) \times 100$ \\
\hline Case 3 & $A^{*}=A^{1 / 2}$ & $B^{*}=B^{1 / 2}$ or $B$ & $\left(1-\sum_{k=1}^{r}\left|b_{k l}^{*}-b_{k}^{\frac{1}{2}}\right| / r\right) \times 100$ or $\left(1-\sum_{k=1}^{r}\left|b_{k l}^{*}-b_{k}\right| / r\right) \times 100$ \\
\hline Case 4 & $A^{*}=1-A$ & $B^{*}=1-B$ & $\left(1-\sum_{k=1}^{r}\left|b_{k l}^{*}-\left(1-b_{k}\right)\right| / r\right) \times 100$ \\
\hline Case 5 & $A^{*}=$ s.t.A & $B^{*}=$ s.t.B & $\left(1-\sum_{k=1}^{r} \mid b_{k l}^{*}-\right.$ s.t. $\left.b_{k} \mid / r\right) \times 100$ \\
\hline FMT & $y$ is $B^{*}[5,18]$ & $x$ is $A^{*}[5,18]$ & New Reductive property criterion $R P C F_{F M T}$ of $x$ is $A^{*},(\%)$ \\
\hline Case 6 & $B^{*}=1-B$ & $A^{*}=1-A$ & $\left(1-\sum_{k=1}^{r}\left|a_{k l}^{*}-\left(1-a_{k}\right)\right| / r\right) \times 100$ \\
\hline Case 7 & $B^{*}=1-B^{2}$ & $A^{*}=1-A^{2}$ or $1-A$ & $\left(1-\sum_{k=1}^{r}\left|a_{k l}^{*}-\left(1-a_{k}\right)^{2}\right| / r\right) \times 100$, or $\quad\left(1-\sum_{k=1}^{r}\left|a_{k l}^{*}-\left(1-a_{k}\right)\right| / r\right) \times 100$ \\
\hline Case 8 & $B^{*}=1-B^{1 / 2}$ & $A^{*}=1-A^{1 / 2}$ or $1-A$ & $\left(1-\sum_{k=1}^{r}\left|a_{k l}^{*}-\left(1-a_{k}\right)^{1 / 2}\right| / r\right) \times 100$, or $\left(1-\sum_{k=1}^{r}\left|a_{k l}^{*}-\left(1-a_{k}\right)\right| / r\right) \times 100$ \\
\hline Case 9 & $B^{*}=B$ & $A^{*}=A$ & $\left(1-\sum_{k=1}^{r}\left|a_{k l}^{*}-a_{k}\right| / r\right) \times 100$ \\
\hline Case 10 & $B^{*}=$ s.t.B & $A^{*}=$ s.t.A & $\left(1-\sum_{k=1}^{r} \mid a_{k l}^{*}-\right.$ s.t. $\left.a_{k} \mid / r\right) \times 100$ \\
\hline
\end{tabular}

In Table 1 Class 1 and Class 2 are as follows.

- Class 1; Case 1, 2, 3, and 4 for FMP, and Case $6,7,8$, and 9 for FMT.

- Class 2; Case 1, 2, 3, and 5 for FMP, and Case 6, 7, 8, and 10 for FMT.

Since FMT is opposite to FMP, Case 1 corresponds to Case 6, Case 2 to Case 7, Case 3 to Case 8, Case 4 to Case 9, and Case 5 to Case 10, respectively. The criterion function for reductive property can be defined as the difference between the consequent of fuzzy rule and conclusion of the fuzzy reasoning. For this, several concepts based on the Table 1 are defined as follows.

Definition 3.1 Let fuzzy sets $A \in F(X), A_{l}^{*} \in F(X)$, $B \in F(Y)$ and $B_{l}^{*} \in F(Y),(l=1,2, \cdots, s k=1,2, \ldots, r)$, for FMP be their antecedent vectors $A=\left[a_{1}, a_{2}, \ldots, a_{k}, \ldots, a_{r}\right]$, the given premise vector $A_{l}^{*}=\left[a_{1 l}^{*}, a_{2 l}^{*}, \ldots, a_{k l}^{*}, \ldots, a_{r l}^{*}\right]$, and the consequent vector $B=\left[b_{1}, b_{2}, \ldots, b_{k}, \ldots, b_{r}\right]$. And then let the fuzzy reasoning conclusion be $B_{l}^{*}=\left[b_{1 l}^{*}, b_{2 l}^{*}, \ldots, b_{k l}^{*}, \ldots, b_{r l}^{*}\right]$. Then the error $E\left(B_{l}^{*}, B\right)$ between the conclusion $B_{l}^{*}$ and consequent $B$, and the error $e\left(A_{l}^{*}, A\right)$ between the given premise $A_{l}^{*}$ and the antecedent $A$ are defined as follows, respectively.
$E\left(B_{l}^{*}, B\right)=\left[b_{1 l}^{*}, b_{2 l}^{*}, \ldots, b_{k l}^{*}, \ldots, b_{r l}^{*}\right]-\left[b_{1}, b_{2}, \ldots, b_{k}, \ldots, b_{r}\right](9)$ $e\left(A_{l}^{*}, A\right)=\left[a_{1 l}^{*}, a_{2 l}^{*}, \ldots, a_{k l}^{*}, \ldots, a_{r l}^{*}\right]-\left[a_{1}, a_{2}, \ldots, a_{k}, \ldots, a_{r}\right]$

Remark 3.1 In Definition 3.1, let us fuzzy sets $A \in F(X), A_{l}^{*} \in F(X) \quad, \quad B \in F(Y) \quad$ and $\quad B_{l}^{*} \in F(Y)$, $(l=1,2, \cdots, s, k=1,2, \ldots, r)$. These fuzzy sets are called normal fuzzy sets in case that their vectors satisfy the following conditions:

$$
\begin{aligned}
& A=\left[a_{1}, a_{2}, \ldots, a_{k}, \ldots, a_{r}\right] \in[0,1], \\
& A_{l}^{*}=\left[a_{1 l}^{*}, a_{2 l}^{*}, \ldots, a_{k l}^{*}, \ldots, a_{r l}^{*}\right] \in[0,1], \\
& B=\left[b_{1}, b_{2}, \ldots, b_{k}, \ldots, b_{r}\right] \in[0,1], \\
& B_{l}^{*}=\left[b_{1 l}^{*}, b_{2 l}^{*}, \ldots, b_{k l}^{*}, \ldots, b_{r l}^{*}\right] \in[0,1],
\end{aligned}
$$

according to [18,31]. For an example of fuzzy sets, $A(x)=\left[\begin{array}{lllll}1, & 0.3, & 0, & 0, & 0\end{array}\right], \quad B(y)=\left[\begin{array}{llll}0, & 0, & 0, & 0.3,\end{array}\right]$ are normal fuzzy sets. In other words normal fuzzy set should include 0 and 1 . In this paper we deal with normal fuzzy sets mentioned above.

Remark 3.2 Unlike Remark 3.1, fuzzy sets are called non-normal fuzzy sets in case that their vectors satisfy the following conditions:

$$
\begin{aligned}
& A=\left[a_{1}, a_{2}, \ldots, a_{k}, \ldots, a_{r}\right] \in(0,1] \text { or } \in[0,1) \text { or } \in(0,1), \\
& A_{l}^{*}=\left[a_{1 l}^{*}, a_{2 l}^{*}, \ldots, a_{k l}^{*}, \ldots, a_{r l}^{*}\right] \in(0,1] \text { or } \in[0,1) \text { or } \in(0,1) \\
& B=\left[b_{1}, b_{2}, \ldots, b_{k}, \ldots, b_{r}\right] \in(0,1] \text { or } \in[0,1) \text { or } \in(0,1) \\
& B_{l}^{*}=\left[b_{1 l}^{*}, b_{2 l}^{*}, \ldots, b_{k l}^{*}, \ldots, b_{r l}^{*}\right] \in(0,1] \text { or } \in[0,1) \text { or } \in(0,1) .
\end{aligned}
$$

For example, $A(x)=\left[\begin{array}{lllll}1, & 0.3, & 0.2, & 0.1, & 0.1\end{array}\right]$ and $B(y)=\left[\begin{array}{lllll}0.2, & 0 . .4,0.5,0.7,1\end{array}\right]$ are non-normal fuzzy sets. In other words, non-normal fuzzy set does not include 0 or 1 . 
Remark 3.3 In real world, when fuzzy sets are applied, engineers and designers generally use normal fuzzy sets. In this paper we do not deal with non-normal fuzzy sets.

Definition 3.2 This Definition 3.2 is to generalize of the criterion for FMP shown in Table 1 according to $[2,4,21-23]$. The 1 th reductive property criterion function $R_{P C F_{F M P-F R-I}^{l}}$ for the Case $1(1=1,2,3,4$, and 5, from Table 1) in FMP can be illustratively defined as equation (10).

$$
\begin{aligned}
& R P C F_{F M P-F R-I}^{l}= \\
& =\left\{\begin{array}{l}
\left(1-\sum_{k=1}^{r}\left|b_{k l}^{*}-b_{k}\right| / r\right) \times 100, \quad \text { for Case } 1 \\
\left(1-\sum_{k=1}^{r}\left|b_{k l}^{*}-b_{k}\right| / r\right) \times 100, \text { or } \\
\left(1-\sum_{k=1}^{r}\left|b_{k l}^{*}-b_{k}^{2}\right| / r\right) \times 100, \text { for } \text { Case } 2 \\
\left(1-\sum_{k=1}^{r}\left|b_{k l}^{*}-b_{k}\right| / r\right) \times 100, \text { or } \\
\left(1-\sum_{k=1}^{r}\left|b_{k l}^{*}-b_{k}^{\frac{1}{2}}\right| / r\right) \times 100, \text { for Case } 3 \\
\left(1-\sum_{k=1}^{r}\left|b_{k l}^{*}-\left(1-b_{k}\right)\right| / r\right) \times 100, \text { for Case } 4 \\
\left(1-\sum_{k=1}^{r} \mid b_{k l}^{*}-\text { s.t. } b_{k} \mid / r\right) \times 100, \text { for Case } 5 \\
\text { s.t. } b_{k}=\text { slightly tiltied of } b_{k}
\end{array}\right.
\end{aligned}
$$

In Case 5 the given premise is $A^{*}=$ s.t.A, and conclusion $B^{*}=$ s.t. $B$. Definition 3.2 is a criterion function based on the Table 1 obtained by $[5,18]$.

Definition 3.3 The reductive property criterion function $R P C F_{F M P-F R}$ for FMP of a fuzzy reasoning method (or algorithm) is defined as follows.

$$
R P C F_{F M P-F R}=\frac{1}{S} \sum_{l=1}^{S} R P C F_{F M P-F R}^{l},(\%)
$$

Remark 3.4 According to Definition 3.3 and Table 1, Class 1 contains Case 1, 2, 3, and 4 for FMP, and Case 6, 7, 8, and 9 for FMT, and then Class 2 contains Case 1, 2, 3, and 5 for FMP, Case $6,7,8$, and 10, for FMT, therefore $s$ is 4 in equation (11).

Definition 3.4 Since FMT is opposite to FMP, let us consider the equation (3) instead of equation (2) for FMT. Now let fuzzy sets $\bar{B} \in F(Y), B_{l}^{*} \in F(Y)$, and $\quad \bar{A} \in F(X) \quad$ be antecedent vectors $\bar{B}=\left[1-b_{1}, 1-b_{2}, \ldots, \quad 1-b_{k}, \ldots, 1-b_{r}\right]$, the given premise vector $B_{l}^{*}=\left[b_{1 l}^{*}, b_{2 l}^{*}, \ldots, b_{k l}^{*}, \ldots, b_{r l}^{*}\right]$, and the consequent vector $\bar{A}=\left[1-a_{1}, 1-a_{2}, \ldots, 1-a_{k}, \ldots, 1-a_{r}\right]$ of fuzzy rule. And the conclusion $A_{l}^{*} \in F(X)$ be $A_{l}^{*}=\left[a_{1 l}^{*}, a_{2 l}^{*}, \ldots, a_{k l}^{*}, \ldots, a_{r l}^{*}\right] \quad(l=1,2, \cdots, s, \quad k=1,2, \ldots, r)$.
Then for FMT the error $E\left(A_{l}^{*}, \bar{A}\right)$ between the fuzzy reasoning conclusion $A_{l}^{*}$ and consequent $\bar{A}$ of fuzzy rule, and the error $e\left(B_{l}^{*}, \bar{B}\right)$ between the given premise $B_{l}^{*}$ and their antecedent $\bar{B}$ are defined as follows, respectively.

$\begin{aligned} E\left(A_{l}^{*}, \bar{A}\right) & =\left[a_{1 l}^{*}, a_{2 l}^{*}, \ldots, a_{k l}^{*}, \ldots, a_{r l}^{*}\right]-\left[1-a_{1}, 1-a_{2}, \ldots, 1-a_{k}, \ldots, 1-a_{r}\right] \\ e\left(B_{l}^{*}, \bar{B}\right) & =\left[b_{1 l}^{*}, b_{2 l}^{*}, \ldots, b_{k l}^{*}, \ldots, b_{r l}^{*}\right]-\left[1-b_{1}, 1-b_{2}, \ldots, 1-b_{k}, \ldots, 1-b_{r}\right]\end{aligned}$

Definition 3.5 The lth reductive property criterion function $R P C F_{F M T-F R-I}^{l}$ for the Case $1(1=6,7,8,9$, and 10, from Table 1) in FMT can be illustratively defined as follows.

$$
\begin{aligned}
& R P C F_{F M T-F R-I}^{l}= \\
& \left\{\begin{array}{l}
\left(1-\sum_{k=1}^{r}\left|a_{k l}^{*}-\left(1-a_{k}\right)\right| / r\right) \times 100, \quad \text { for Case } 6 \\
\left(1-\sum_{k=1}^{r}\left|a_{k l}^{*}-\left(1-a_{k}\right)^{2}\right| / r\right) \times 100, \quad \text { or } \\
\left(1-\sum_{k=1}^{r}\left|a_{k}^{r}-\left(1-a_{k}\right)\right| / r\right) \times 100, \quad \text { for Case } 7
\end{array}\right. \\
& \begin{cases}\left(1-\sum_{k=1}^{r}\left|a_{k l}^{*}-\left(1-a_{k}\right)\right| / r\right) \times 100, & \text { for Case } 7 \\
\left(1-\sum_{k=1}^{r}\left|a_{k l}^{*}-\left(1-a_{k}\right)^{\frac{1}{2}}\right| / r\right) \times 100, & \text { or }\end{cases} \\
& =\left\{\left(1-\sum_{k=1}^{r}\left|a_{k l}^{*}-\left(1-a_{k}\right)^{\frac{1}{2}}\right| / r\right) \times 100,\right. \text { or } \\
& \left(1-\sum_{k=1}^{r}\left|a_{k l}^{*}-\left(1-a_{k}\right)\right| / r\right) \times 100, \quad \text { for Case } 8 \\
& \left(1-\sum_{k=1}^{r}\left|a_{k l}^{*}-a_{k}\right| / r\right) \times 100, \quad \text { for Case } 9 \\
& \left(1-\sum_{k=1}^{r} \mid a_{k l}^{*}-\text { s.t. } a_{k} \mid / r\right) \times 100, \quad \text { for Case } 10 \\
& \text { s.t. } a_{k}=\text { slightly tiltied of } a_{k}
\end{aligned}
$$

In Case 10 the given premise is $B^{*}=$ slightly tilted of $B=$ s.t. $B$, Conclusion $A^{*}=$ slightly tilted of $A=$ s.t. $A$.

Definition 3.5 is also a criterion function based on the Table 1 obtained by $[5,18]$.

Definition 3.6 The reductive property criterion function $R P C F_{F M T-F R}$ for FMT are defined as follows.

$$
R P C F_{F M T-F R}=\frac{1}{S} \sum_{l=1}^{s} R P C F_{F M T-F R}^{l},(\%)
$$

The reductive property of fuzzy reasoning can be considered as the reductive property of a fuzzy reasoning method or algorithm $=$ average of (reductive property for FMP and reductive property for FMT).

Definition 3.7 The criterion function for checking of the reductive property of fuzzy reasoning method is defined as arithmetic average value of $R P C F_{F M P-F R}$ and $R P C F_{F M T-F R}$.

$$
R P C F_{F R}=\frac{1}{2}\left(R P C F_{F M P-F R}+R P C F_{F M T-F R}\right) \text {, }
$$

Remark 3.5 In equation (13)-(15), indexes are the same as equation (10)-(11). According to above 
two definition, when the reduction property criterion function $R P C F_{F M P-F R}=100(\%)$ and $R P C F_{F M T-F R}$ $=100(\%)$, then the reductive property of fuzzy reasoning method (or algorithm) is completely satisfied. This means that the given consequent vector(resp. the given antecedent vector) is equal to fuzzy reasoning result vector, that is, $b_{k}^{*}=b_{k}, k=1,2, \ldots, r \quad$, i.e., $\quad B^{*}=B \quad, \quad$ (resp. $a_{k}^{*}=a_{k}, k=1,2, \ldots, r$, i.e., $A^{*}=A$ ), for FMP (resp. FMT). In other words, the larger $R P C F_{F M P-F R}$ (resp. $\left.R P C F_{F M T-F R}\right)$ is, the more the result of fuzzy reasoning satisfies the reductive property, and the smaller $R P C F_{\text {MP }-F}$ (resp. $R P C F_{F M T-F R}$ ) is, the less it satisfies. At worst, when criterion function $R P C F_{F M P-F R}=0(\%)$ and $R P C F_{F M T-F K}=0(\%)$, then the fuzzy reasoning method does not completely satisfy. Therefore the reductive property criterion function about every reasoning method in the fuzzy systems satisfies $0 \leq R P C F_{F M P-F R} \leq 100, \quad 0 \leq R P C F_{F M T-F R} \leq 100$ for FMP and FMT, respectively. These definitions differ largely from the several previous ones $[2,4,8]$. Therefore according to our definition method the fuzzy reasoning result can be more correctly evaluated, and effectively used in a lot of the practical problems.

Now let us discuss checking for the reductive property of fuzzy reasoning method.

Example 3.1 Assume that the fuzzy sets of the rule are given as $A(x)=\left[\begin{array}{lllll}1, & 0.3, & 0, & 0, & 0\end{array}\right]$, $B(y)=\left[\begin{array}{lllll}0, & 0 & 0 & 0.3, & 1\end{array}\right]$, and the given premise for FMP $A^{*}(x)=A(x)=\left[\begin{array}{lllll}1, & 0.3, & 0, & 0, & 0\end{array}\right]$, the premise for FMT $B^{*}(y)=B(y)=\left[\begin{array}{lllll}0, & 0, & 0, & 0.3,1\end{array}\right]$, then the new conclusion reasoning result by any fuzzy reasoning method (for instance WW) is obtained as $B^{*}(y) \neq B(y)=\left[\begin{array}{llll}0, & 0 & 0.1 & 0.4,1\end{array}\right]$ for $\mathrm{FMP}, A^{*}(x) \neq A(x)=\left[\begin{array}{lllll}1, & 0.7, & 0.4, & 0.1, & 0\end{array}\right]$ for $\mathrm{FMT}$, respectively. At this time according to our new method, the criterion function is calculated as $R P C F_{W W}=\frac{1}{2}\left(R P C F_{F M P-W W-I}+R P C F_{F M T-W W-I}\right)=\frac{1}{2}(96+38=67(\%)$.

Consequently, the reductive property of a fuzzy reasoning method WW is satisfied as $67(\%)$. But according to [2], since $B^{*}(y) \neq B(y)$ for FMP and $A^{*}(x) \neq A(x)$ for FMT, the reductive property of a fuzzy reasoning method WW is not satisfied as $0(\%)$, thus their evaluation is strict and not right.

Example 3.2 Let us consider the reductive property of Example 1 and 2 in [7]. The fuzzy sets of the rule are as $[$ small $]=\left[\begin{array}{lllll}1, & 0.3, & 0, & 0, & 0\end{array}\right]$, [large $]=\left[\begin{array}{lllll}0, & 0, & 0 & 0.3,1\end{array}\right]$ and the premise $[$ medium $]=\left[\begin{array}{lllll}0, & 0.3, & 1 & 0.3, & 0\end{array}\right]$. The conclusions by CRI

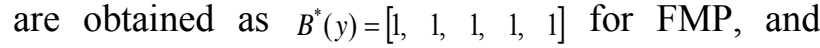
$A^{*}(x)=[0.3,0.7,1,1,1]$ for FMT. According to our Definition 3.3, 3.6, and 3.7, the reductive property of a fuzzy reasoning method $\mathrm{WW}$ is as follows.

$$
\begin{aligned}
R P C F_{W W} & =\frac{1}{2}\left(R P C F_{F M P-W W-I}+R P C F_{F M T-W W-I}\right)= \\
& =\frac{1}{2}(43.27+89.5)=66.385(\%)
\end{aligned}
$$

Now let us consider the difference of ours and [17]'s checking method. [17]'s checking method is very strict and has some weakness. [16]'s weakness is as follows. For the same Case such as Example 3.1, according to [17]'s checking method, reasoning result are $B^{*}(y)=\left[\begin{array}{lllll}0, & 0, & 0.1, & 0.4, & 1\end{array}\right] \neq B(y)=\left[\begin{array}{lllll}0, & 0, & 0, & 0.3, & 1\end{array}\right]$ for FMP, and $A^{*}(x)=\left[\begin{array}{lllll}1, & 0.7, & 0.4, & 0.1, & 0\end{array}\right]$ $\neq A(x)=\left[\begin{array}{lllll}1, & 0.3, & 0, & 0, & 0\end{array}\right]$ for FMT, respectively. Then the reductive property of the fuzzy reasoning method WW is not satisfied by the criterion of Table 1, that is, it is not flexible and soft. For this evaluation, considering by [17]'s viewpoint, it is satisfied as $0(\%)$ or is not satisfied as $100(\%)$, vice versa. So in order to overcome [17]'s weakness, we generalized and extended the criterion for FMP and FMT shown in Table 1 according to [17]. Frankly speaking, even though reasoning results are obtained as $B^{*}(y) \neq B(y)$ for FMP, and $A^{*}(x) \neq A(x)$ for FMT, respectively, our checking method can discuss the degree of coincidence between the given premise and the antecedent of fuzzy rule. In other words our proposed criterion function (15) tries to calculate the percentage degree of coincidence between the consequent $B(y)$ (resp. $A(x)$ ) of fuzzy rule and the conclusion $B^{*}(y)$ (resp. $A^{*}(x)$ ) of the reasoning, and then calculate the average of 2 percentage degrees of coincidence for FMP and FMT.

Remark 3.6 The higher the degree of coincidence between $B(y)$ (resp. $A(x)$ ) and $B^{*}(y)$ $\left(\operatorname{resp} . A^{*}(x)\right)$ is, the better the reductive property of FMP (resp. FMT) is. Therefore as shown in Example 3.1 and 3.2, our new checking method of the reductive property is softer and better in accordance with general human understanding and practical problems than [17]'s one.

\subsection{New Fuzzy Reasoning Method For FMP}

In this subsection we define several concepts and equation about new FMP-DM method based on distance measure. The news in this subsection are that; (1) according to definition of difference vector, discrete sign vector, Euclidian distance measure, quasi-fuzzy reasoning result, maximum and minimum of the quasi-fuzzy reasoning result, the fuzzy reasoning conclusion result for solving FMP based on distance measure is proposed, (2) 
according to Principle for solving of FMP-DM Problem, new theorem for FMP-DM solution is proposed and proved.

According to the paper [25], distance measure is as follows. Let $F_{0}(R)$ be all continuous fuzzy subsets of $R$ whose $\alpha$-cuts are always bounded intervals. These will be called fuzzy numbers and are the fuzzy sets most widely used in practical applications. We need to be able to compute the distance between any fuzzy set $A$ and $B$ in $F_{0}(R)$. We know how to find the distance between two real numbers $x, y$. The distance is $|x-y|=D M(x, y)$. We also know how to find the distance between two points in $R^{2}$. The function $\operatorname{DM}(x, y)$ used to compute distance is called a distance measure (DM). The basic properties of $\mathrm{DM}$, i.e., $\operatorname{DM}(x, y)$ for every $x, y$ in real space $R$ are:

- $D M(x, y) \geq 0$; i.e., distance is not negative;

- $D M(x, y)=D M(y, x)$; i.e., distance is symmetric;

- $D M(x, y)=0$; if and only if $x=y$; i.e., we get zero distance only when $x=y$.

- $D M(x, y) \leq D M(x, z)+D M(z, y)$; i.e., it is shorter to go directly from $\mathrm{x}$ to $\mathrm{y}$ instead of first going to intermediate point $\mathrm{z}$.

Definition 3.8 Let the antecedent $A$ and the given premises $A_{l}^{*}$ for FMP be their discrete vector $A=\left[a_{1}, a_{2}, \ldots, a_{k}, \ldots, a_{r}\right], A_{l}^{*}=\left[a_{1 l}^{*}, a_{2 l}^{*}, \ldots, a_{k l}^{*}, \ldots, a_{r l}^{*}\right]$, respectively, where $a_{k}, a_{k l}^{*}$ are individual element of $A, A_{l}^{*}$, which are membership values in its fuzzy set, respectively. For FMP the individual elements $\alpha_{k l}$ of difference vector $\alpha_{l}=\left[\alpha_{1 l}, \alpha_{2 l}, \cdots, \alpha_{k l}, \cdots, \alpha_{r l}\right]$, $(k=1,2, \ldots, r, l=1,2, \ldots, S)$, are defined as follows.

$$
\alpha_{k l}=a_{k l}^{*}-a_{k} \text {, for FMP }
$$

Definition 3.9 Let a discrete sign vector be $p_{l}=\left[p_{l l}, p_{2 l}, \cdots, p_{k l}, \cdots, p_{r l}\right],(l=1,2, \cdots, s)$. Then element $p_{k l}$ of the sign vector is defined by two ways, i.e., $P(+1,0,-1)$ form and $P(+1,-1)$ form, for FMP, as following equation, respectively.

$$
\begin{aligned}
& P(+1,0,-1) \text { form } P_{k l}=\operatorname{sign}\left(\alpha_{k l}\right)= \begin{cases}+1, & \alpha_{k l}>0 \\
0, & \alpha_{k l}=0 \\
-1, & \alpha_{k l}<0\end{cases} \\
& P(+1,-1) \text { form } \quad P_{k l}=\operatorname{sign}\left(\alpha_{k l}\right)= \begin{cases}+1, & \alpha_{k l} \geq 0 \\
-1, & \alpha_{k l}<0\end{cases}
\end{aligned}
$$

Definition 3.10 (See [21,22]) For FMP, the distance measure $\operatorname{DM}\left(A_{l}^{*}, A\right)$ between the antecedent fuzzy set $A$ and the given premise $A_{l}^{*}$ by using Euclidian distance measure is defined as follows.

$$
D M\left(A_{l}^{*}, A\right)=\left[\sum_{k=1}^{r}\left[a_{k l}^{*}-a_{k}\right]^{2} / r\right]^{1 / 2}, \text { for FMP }
$$

Definition 3.11 The quasi-fuzzy reasoning result $\widetilde{B}_{l}$ for FMP can be defined as follows.

$$
\widetilde{B}_{l}=\left\{\begin{array}{lc}
B+D M\left(A_{l}^{*}, A\right) \times P_{l}, & \text { if } \\
1-B+D M\left(A_{l}^{*}, A\right) \times P_{l}, & \text { if Case } 1,2, \text { and } 3 \\
\text { s.t. } B+D M\left(A_{l}^{*}, A\right) \times P_{l}, & \text { if Case } 5
\end{array}\right.
$$

Definition 3.12 The maximum $\xi_{l}$ and minimum $\eta_{l}$ of the quasi-fuzzy reasoning result $\widetilde{B}_{l}$ are defined as follows, respectively.

$$
\xi_{l}=\max _{1 \leq k \leq r} \widetilde{B}_{l}, \quad \eta_{l}=\min _{1 \leq k \leq r} \widetilde{B}_{l} \quad \text { for FMP }
$$

Definition 3.13 The fuzzy reasoning conclusion result for solving FMP based on DM can be defined as equation (22), in this paper.

$$
\text { (FMP-DM) } \quad B_{l}^{*}=\frac{\widetilde{B}_{l}-\eta_{l}}{\xi_{l}-\eta_{l}}
$$

Where $l=1,2, \cdots, s$ is index of the given premises $A_{l}^{*}$ for FMP, that is, $B_{l}^{*}$ is fuzzy reasoning conclusion by the 1 th given premise $A_{l}^{*}$ for FMP. And $\Phi \in F(X)$ is an empty set, $X$ is universe of discourse, and $x \in X, A \in F(X)$. Here, $A_{l}^{*}, \widetilde{A}_{l}$ and $A$ are the fuzzy sets in $F(X)$. The equation (22) is an standardization expression of the quasi-fuzzy reasoning result $\widetilde{B}_{l}$ for FMP. The proposed method expressed by equation (22) is called distance measure method for the FMP with single input single output fuzzy system in this paper, for short FMP-DM. When combined $B_{l}^{*}$ and $A_{l}^{*}$, the fuzzy reasoning conclusion $B^{*}$ for FMP-DM can be described as follows.

$$
B^{*}=\bigcup_{l=1}^{s} B_{l}^{*}, \quad \text { for FMP-DM }
$$

Where $U$ is not max, but means the union of individual fuzzy sets obtained by fuzzy reasoning for FMP. Consequently, as defined in subsection 3.2, the criterion function $R P C F_{F R}$ for checking of the reductive property of fuzzy reasoning method is reflecting the degree of consistency between consequent $B$ and conclusion $B^{*}$ by equation (23), which is based on the degree of consistency between the antecedent $A$ and the given premise $A^{*}$ for FMP. Therefore it can be reasonable to consider the degree of consistency between 
conclusion $B^{*}$ and consequent $B$ to evaluate the reductive property (or reducibility) of FMP with considering the degree of consistency between the antecedent $A$ and the given premise $A^{*}$. For classical 2-valued logic, general modus ponens may be interpreted as if "if $x$ is $A$ then $y$ is $B$ " and " $A^{*}=A$ " then " $B$ " $=B$ ". According to fuzzy logic, we hope to provide logical analysis for fuzzy modus ponens. Based on distance measure, FMP solution can be interpreted as if " if $x$ is $A$ then $y$ is $B$ " and " $A^{*}$ is closer to $A$ " then " $B^{*}$ is closer to $B$ ". From the logical analysis of FMP solution, we can find that the conclusion $B^{*}$ not only relates to $A^{*}$ and " if $x$ is $A$ then $y$ is $B$ ", but also relates to the distance measure of $A^{*}$ and $A$. How to select $D M\left(B^{*}, B\right)$ to make the conclusion of fuzzy reasoning more reasonable? We hope that $D M\left(B^{*}, B\right)$ is equal to $D M\left(A^{*}, A\right)$. And this property is proper with respect to fuzzy reasoning. Our aim is to search the fuzzy sets $B^{*}$ such that the distance measure $D M\left(B^{*}, B\right)$ should be fully supported by distance measure $D M\left(A^{*}, A\right)$. That is, following equation should be satisfied.

$$
D M\left(B^{*}, B\right)=D M\left(A^{*}, A\right) \text {, for FMP }
$$

There are a lot of fuzzy subsets on Y that satisfy the equation (1). We hope the fuzzy subset as the conclusion of fuzzy reasoning satisfying the reductive property to be selected as correctly as possible.

Principle for Solving of FMP-DM Problem. The FMP-DM conclusion $B^{*}$ of equation (1) for a distance measure is the fuzzy subset of $Y$ satisfying equation (24).

According to this principle, theorem of FMP-DM is as follows.

Theorem 3.1 Assume that distance measure is Euclidean metric, then the FMP-DM solution of the equation (1) satisfying the equation (24) is described as follows.

$$
B^{*}=\left\{\begin{array}{lc}
f\left(B+D M\left(B^{*}, B\right)\right), \text { if } & \text { Case } 1,2, \text { and } 3 \\
f\left(1-B+D M\left(B^{*}, B\right)\right), & \text { if Case } 4 \\
f\left(\text { s.t. } B+D M\left(B^{*}, B\right)\right), & \text { if Case } 5
\end{array}\right.
$$

, where $f$ is standardization operator. Therefore there is no information loss of the fuzzy reasoning processing by $f$.

\section{Proof.}

( i ) Let's consider for Case 1, Case 2, and Case 3. For FMP-DM it is evident that if $A_{l}^{*} \cap A=\Phi$ then $B^{*}=0$. When $A_{l}^{*} \cap A \neq \Phi$ then the fuzzy reasoning conclusion for FMP is obtained as follows.

$$
\begin{aligned}
B^{*}= & \bigcup_{l=1}^{s} B_{l}^{*}=B_{1}^{*} \cup B_{2}^{*} \cup \cdots B_{l}^{*} \cup \cdots B_{s}^{*}=\left(\widetilde{B}_{1}-\eta_{1}\right) /\left(\xi_{1}-\eta_{1}\right) \cup \\
& \left(\widetilde{B}_{2}-\eta_{2}\right) /\left(\xi_{2}-\eta_{2}\right) \cup \cdots \cup\left(\widetilde{B}_{l}-\eta_{l}\right) /\left(\xi_{l}-\eta_{l}\right) \cup \cdots \cup\left(\widetilde{B}_{s}-\eta_{s}\right) /\left(\xi_{s}-\eta_{s}\right) \\
= & \left(B_{1}+D M\left(A_{l}^{*}, A\right) \times P_{1}-\eta_{1}\right) /\left(\xi_{1}-\eta_{1}\right) \cup\left(B_{2}+D M\left(A_{2}^{*}, A\right)\right. \\
& \left.\times P_{2}-\eta_{2}\right) /\left(\xi_{2}-\eta_{2}\right) \cup \cdots, \cup\left(B_{l}+D M\left(A_{l}^{*}, A\right) \times P_{l}-\eta_{l}\right) /\left(\xi_{l}-\eta_{l}\right) \\
& \cup \cdots \cup\left(B_{s}+D M\left(A_{s}^{*}, A\right) \times P_{s}-\eta_{s}\right) /\left(\xi_{s}-\eta_{s}\right) \\
= & \left(B_{1} \cup B_{2} \cup \cdots \cup B_{l} \cup \cdots B_{s}\right)+\left(D M\left(A_{l}^{*}, A\right) \times P_{1}-\eta_{1}\right) /\left(\xi_{1}-\eta_{1}\right) \cup D M\left(A_{2}^{*}, A\right) \\
& \left.\left.\times P_{2}-\eta_{2}\right) /\left(\xi_{2}-\eta_{2}\right) \cup \cdots \cup D M\left(A_{l}^{*}, A\right) \times P_{l}-\eta_{l}\right) /\left(\xi_{l}-\eta_{l}\right) \cup \cdots \\
& \left.\left.\cup D M\left(A_{s}^{*}, A\right) \times P_{s}-\eta_{s}\right) /\left(\xi_{s}-\eta_{s}\right)\right) \\
= & \left.\bigcup_{l=1}^{s} B_{l}+\bigcup_{l=1}^{s} D M\left(A_{l}^{*}, A\right) \times P_{l}-\eta_{l}\right) /\left(\xi_{l}-\eta_{l}\right) \\
= & \bigcup_{l=1}^{s} B_{l}+\left(\bigcup_{l=1}^{s} f\left(D M\left(A_{l}^{*}, A\right)\right)=\bigcup_{l=1}^{s} B_{l}+f\left(\bigcup_{l=1}^{s} D M\left(A_{l}^{*}, A\right)\right)\right. \\
= & f\left(B+D M\left(A^{*}, A\right)\right)=f\left(B+D M\left(B^{*}, B\right)\right)
\end{aligned}
$$

(ii) Let us consider for Case 4. The proof of ( ii ) is similar to ( $i$ ), so it is abbreviated.

(iii) Let us consider for Case 5. The proof of ( iii) is also similar to ( $i$ ), thus it is also abbreviated.

Thus we have proved that fuzzy reasoning conclusion $B^{*}$ for FMP-DM obtained by the equation (25) satisfies the equation (24). The information loss is guaranteed by maximum $\xi_{l}$ and minimum $\eta_{l}$ of quasi-reasoning result $\widetilde{B}_{l}$ in above equation for FMP.

Proposed fuzzy reasoning method FMP-DM is as follows.

Step 1; Compute difference vector between the antecedent $A$ and the given premises $A_{l}^{*}$ according to (16).

Step 2; Compute the sign vector is defined by $P(+1,0,-1)$ form and $P(+1,-1)$ form according to the equation (17), (18).

Step 3; Compute the distance measure $\operatorname{DM}\left(A_{l}^{*}, A\right)$ between the antecedent fuzzy set $A$ and the given premise $A_{l}^{*}$ according to (19).

Step 4; Compute maximum and minimum for quasi-fuzzy reasoning result $\widetilde{B}_{l}$ for FMP according to the equation (20).

Step 5; Compute the quasi-fuzzy reasoning result $\widetilde{B}_{l}$ according to the equation (21).

Step 6; Obtain fuzzy reasoning conclusion result for solving FMP according to the equation (22).

Below an example for FMP-DM is shown.

Example 3.3 Let us consider for FMP-DM in Class 1. According to distance measure for FMP, FMP-DM can be obtained as the equation (22). For the antecedent $A=[1,0.3,0,0,0]$, the consequent $B=[0,0,0,0.3,1]$, for four Cases, fuzzy reasoning results are as follows. In Case 1, the given premise is $A^{*}=A$, distance measure $D M$ is calculated as 
$D M=0$, quasi-reasoning result $\widetilde{B}$ for FMP-DM is calculated as $\widetilde{B}=[0,0,0,0.31] \quad, \quad$ since $B^{*}=\widetilde{B}=[0,0,0,0.3,1]=B$, therefore the reductive property is $100(\%)$. In Case 2, 3, and 4 , the reductive property is calculated as $91.16(\%), 92.83$ $(\%)$, and $68.25(\%)$, respectively. Thus total reductive property criterion function value for FMP-DM presented in this paper is obtained as $R P C F_{F M P-D M}=87.7285$ (\%).

Example 3.4 Let us consider another example for FMP-DM in Class 1. For example, let the fuzzy rule is "if $x$ is $A$ then $y$ is $B$ ", consequent $B=\left[\begin{array}{lll}0.2 & 0.4 & 0.5\end{array}\right.$ $0.7,1]$, the quasi-reasoning result $\beta=B, \xi=1$ and $\eta$ $=0.2$ when the premise is " $x$ is $A$ ". It's obviously that the reasoning result $B^{*}$ is not equal to $B . A$ lot of detailed calculations are carried out in this article, but full text entirely based on two examples in reference paper.

\subsection{New Fuzzy Reasoning Method For FMT}

The news in this subsection are as follows; (1) according to definition of difference vector, discrete sign vector, Euclidian distance measure, quasi-fuzzy reasoning result, maximum and minimum of the quasi-fuzzy reasoning result, the fuzzy reasoning conclusion result for solving FMT based on distance measure is proposed, (2) on the basis of new principle for solving of FMT-DM problem, new theorem for FMT-DM solution is proposed. In this subsection we define several concepts and equation about new FMT-DM based on DM in [24].

Definition 3.14 Let the antecedent $B$ and the given premises $B_{l}^{*}$ for FMT be their discrete vector $B=\left[b_{1}, b_{2}, \ldots, b_{k}, \ldots, b_{r}\right]$, and $B_{l}^{*}=\left[b_{1 l}^{*}, b_{2 l}^{*}, \ldots, b_{k l}^{*}, \ldots, b_{r l}^{*}\right]$ , $(k=1,2, \ldots, r)$, respectively. Where $b_{k}$, and $b_{k l}^{*}$ are individual elements of $B$ and $B_{l}^{*}$, which are membership values in its fuzzy set, respectively. For FMT the individual elements $\beta_{k l}$ of difference vector $\beta_{l}=\left[\beta_{1 l}, \beta_{2 l}, \cdots, \beta_{k l}, \cdots, \beta_{r l}\right]$ are defined as follows.

$$
\beta_{k l}=b_{k l}^{*}-b_{k}, \text { for FMT }
$$

Definition 3.15 Let a discrete sign vector be $p_{l}=\left[p_{1 l}, p_{2 l}, \cdots, p_{k l}, \cdots, p_{r l}\right],(l=1,2, \cdots, s) \quad$. Then element $p_{k l}$ of the sign vector is defined by two ways, i.e., $P(+1,0,-1)$ form and $P(+1,-1)$ form, for FMT, as following equations, respectively.

$$
P(+1,0,-1) \text { form } P_{k l}=\operatorname{sign}\left(\beta_{k l}\right)= \begin{cases}+1, & \beta_{k l}>0 \\ 0, & \beta_{k l}=0 \\ -1, & \beta_{k l}<0\end{cases}
$$

$$
P(+1,-1) \text { form } \quad P_{k l}=\operatorname{sign}\left(\beta_{k l}\right)= \begin{cases}+1, & \beta_{k l} \geq 0 \\ -1, & \beta_{k l}<0\end{cases}
$$

Definition 3.16 (See [21,22]) For FMT, the distance measure $D M\left(B_{l}^{*}, B\right)$ between the antecedent fuzzy set $B$ and the given premise $B_{l}^{*}$ by using Euclidian distance measure is defined as follows, according to the paper $[21,22]$.

$$
D M\left(B_{l}^{*}, B\right)=\left[\sum_{k=1}^{r}\left[b_{k l}^{*}-b_{k}\right]^{2} / r\right]^{1 / 2} \text {, for FMT }
$$

Definition 3.17 The quasi-fuzzy reasoning result $\widetilde{A}_{l}$ for FMT can be defined as follows.

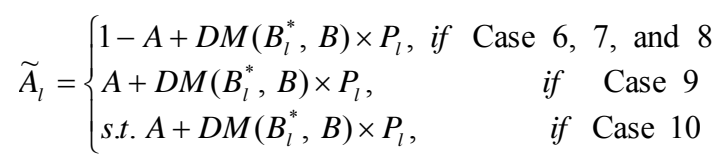

Definition 3.18 The maximum $\xi_{l}$ and minimum $\eta_{l}$ of the quasi-fuzzy approximate reasoning result $\widetilde{A}_{l}$ are defined as follows, respectively.

$$
\xi_{l}=\max _{1 \leq k \leq r} \widetilde{A}_{l}, \quad \eta_{l}=\min _{1 \leq k \leq r} \widetilde{A}_{l} \text { for FMT }
$$

Definition 3.19 The fuzzy reasoning results for solving of fuzzy modus tollens based on DM is defined as equation (32).

$$
\left(\text { FMT-DM) } \quad A_{l}^{*}=\frac{\widetilde{A}_{l}-\eta_{l}}{\xi_{l}-\eta_{l}}\right.
$$

Where $l=1,2, \cdots, s$ is index of the given premises $B_{l}^{*}$ for FMT, that is, $A_{l}^{*}$ is fuzzy reasoning conclusion by the 1 th given premise $B_{l}^{*}$ for FMT. And $\Phi \in F(Y)$ is an empty set for FMT, also $Y$ is universe of discourse, and $y \in Y$, $B \in F(Y)$. Here, $B_{l}^{*}, \widetilde{B}_{l}$, and $B$ are the fuzzy sets in $F(Y)$. The equation (32) is a standardization expression of the quasi-fuzzy reasoning result $\widetilde{A}_{l}$ for FMT. The proposed method expressed by equation (32) is called distance measure method of fuzzy reasoning for FMT with single input single output fuzzy system in this paper, for short FMT-DM. When combined $B_{l}^{*}$ and $A_{l}^{*}$, the fuzzy reasoning conclusion $A^{*}$ for FMT can be described as follows.

$$
A^{*}=\bigcup_{l=1}^{s} A_{l}^{*}, \quad \text { for FMT }
$$

Where $U$ is not max, means the union of individual fuzzy sets obtained by fuzzy reasoning for FMT. Consequently, as defined in subsection 3.1, the criterion function $R P C F_{F R}$ for checking of the reductive property of fuzzy reasoning method is 
reflecting the degree of consistency between consequent $A$ and conclusion $A^{*}$ by equation (32), which is based on the degree of consistency between the antecedent $B$ and the given premise $B^{*}$ for FMT. Therefore it can be reasonable to consider the degree of consistency between the fuzzy reasoning conclusion $A^{*}$ and consequent $A$ to evaluate the reductive property (or reducibility) of FMT with considering the consistency between the antecedent $B$ and the given premise $B^{*}$. For classical 2-valued logic, general modus ponens may be interpreted as if "if $x$ is $A$ then $y$ is $B$ " and $A^{*}=A$ then $B^{*}=B$. According to fuzzy logic, we hope to provide logical analysis for fuzzy modus ponens. Based on distance measure, FMP solution can be interpreted as if "if $x$ is $A$ then $y$ is $B$ " and " $A^{*}$ is closer to $A$ " then " $B^{*}$ is closer to $B$ ". From the logical analysis of FMP solution, we can find that the conclusion $B^{*}$ not only relates to $A^{*}$ and " if $x$ is $A$ then $y$ is $B$ ", but also relates to the distance measure of $A^{*}$ and $A$. How to select $D M\left(B^{*}, B\right)$ to make the conclusion of fuzzy reasoning more reasonable? We hope that $D M\left(B^{*}, B\right)$ is equal to $D M\left(A^{*}, A\right)$. And this property is proper with respect to fuzzy reasoning. Our aim is to search the fuzzy sets $B^{*}$ such that the distance measure $D M\left(B^{*}, B\right)$ should be fully supported by distance measure $D M\left(A^{*}, A\right)$. Let us consider FMT-DM. Here our aim is to search the fuzzy sets $A^{*}$ such that the distance measure $D M\left(A^{*}, \bar{A}\right)$ obtained by the fuzzy reasoning conclusion and the consequent should be fully supported by distance measure $D M\left(B^{*}, \bar{B}\right)$ obtained by the given premise and the antecedent. That is, following equation for FMT should be satisfied.

$$
D M\left(A^{*}, \bar{A}\right)=D M\left(B^{*}, \bar{B}\right) \quad \text {, for FMT }
$$

There are a lot of fuzzy subsets on $X$ that satisfy the equation (3). We try to select the fuzzy subset as the conclusion of fuzzy reasoning satisfying equation (34).

Principle for solving of FMT-DM Problem. The FMT-DM conclusion $A^{*}$ of the equation (3) for a distance measure is the fuzzy subset of $X$ satisfying the equation (34).

According to this principle, FMT-DM method is as follows.

Theorem 3.2 Assume that distance measure is Euclidean metric, then the FMT-DM solution of the equation (3) satisfying the equation (34) is expressed as follows. Where $t$ is standardization operator. Hereby there is no information loss of the fuzzy reasoning processing by $t$.

$$
A^{*}=\left\{\begin{array}{lr}
f\left(\bar{A}+D M\left(A^{*}, A\right)\right), \text { if } & \text { Case } 6,7, \text { and } 8 \\
f\left(A+D M\left(A^{*}, A\right)\right), & \text { if Case } 9 \\
f\left(\text { s.t. } A+D M\left(A^{*}, A\right)\right) & \text { if Case } 10
\end{array}\right.
$$

As known from the equation (25) and (35), FMT is opposite to FMP. So its proof is omitted here.

Proposed fuzzy reasoning method FMT-DM is as follows.

Step 1; Solve difference vector between the antecedent $B$ and the given premises $B_{l}^{*}$ according to the equation (26).

Step 2; Solve the sign vector is defined by $P(+1,0,-1)$ form and $P(+1,-1)$ form according to the equation (27), (28).

Step 3; Solve the distance measure $D M\left(B_{l}^{*}, B\right)$ between the antecedent fuzzy set $B$ and the given premise $B_{l}^{*}$ according to (29).

Step 4; Solve maximum and minimum for quasi-fuzzy reasoning result $\tilde{A}_{l}$ according to the equation (30).

Step 5; Solve the quasi-fuzzy reasoning result $\widetilde{A}_{l}$ according to the equation (31).

Step 6; Decide fuzzy reasoning conclusion result for solving FMT according to the equation (32).

\section{Checking of CRI, TIP, AARS, and Proposed DMM}

The most general forms of the CRI solutions of FMP and FMT are as equation (36) and (37).

$$
\begin{array}{ll}
(\text { FMP-CRI) } & B^{*}(y)=\bigvee_{x \in U}\left(A^{*}(x) \otimes(A(x) \rightarrow B(y))\right) \\
(\text { FMT-CRI) } & \left.A^{*}(x)=\bigvee_{y \in V}\left(B^{*}(y)\right) \otimes(A(x) \rightarrow B(y))\right)
\end{array}
$$

FMP-CRI and FMT-CRI reductive properties based on equation (36) and (37) for Class 1 are shown in Table 2.

The reductive property of FMP-CRI and by Łukasiewicz, Gödel, R0 and Gougen are more than FMT-CRI with respect to [2], respectively.

The general forms of the TIP are as follows. [26]

$$
\begin{aligned}
& \left(\text { FMP-TIP) } \quad B^{*}(y)=\underset{x \in U}{\vee}\left(A^{*}(x) \otimes(A(x) \rightarrow B(y))\right)\right. \\
& \left(\text { FMT-TIP) } \quad A^{*}(x)=\widehat{\bigwedge}_{y \in V}\left((A(x) \rightarrow B(y)) \rightarrow B^{*}(y)\right)\right)
\end{aligned}
$$

Table 2 FMP-CRI and FMT-CRI reductive property in Class 1 


\begin{tabular}{|c|c|c|c|c|}
\hline FMP-CRI & \multicolumn{4}{|c|}{ FMP-CRI-Conclusion $B^{*}(y)$ and Reductive Property } \\
\hline Premise $A^{*}(x)$ & \multicolumn{2}{|c|}{$\begin{array}{c}\text { FMP- CRI -Łukasiewicz } \\
\end{array}$} & \multicolumn{2}{|c|}{ FMP- CRI -Gödel } \\
\hline$[1,0.3,0,0,0]$ & {$[0,0,0,0.3,1]$} & $100 \%$ & {$[0,0,0,0.3,1]$} & $100 \%$ \\
\hline$[1,0.09,0,0,0]$ & {$[0,0,0,0.3,1]$} & $95.8 \%$ & {$[0,0,0,0.3,1]$} & $95.80 \%$ \\
\hline$[1,0.548,0,0,0]$ & {$[0.248,0.248,0.248,0.548,1]$} & $85.14 \%$ & {$[0,0,0,0.548,1]$} & $100 \%$ \\
\hline$[0,0.7,1,1,1]$ & {$[1,1,1,1,1]$} & $74.00 \%$ & {$[1,1,1,1,1]$} & $74.00 \%$ \\
\hline$R P C F_{F M P-C R I-I}$ & \multicolumn{2}{|l|}{$88.73 \%$} & \multicolumn{2}{|c|}{$92.45 \%$} \\
\hline FMT-CRI & \multicolumn{4}{|c|}{ FMT-CRI-Conclusion $A^{*}(x)$ and Reductive Property } \\
\hline Premise $B^{*}(y)$ & \multicolumn{2}{|c|}{ FMT-CRI-Łukasiewicz } & \multicolumn{2}{|c|}{ FMT-CRI-Gödel } \\
\hline$[1,1,1,0.7,0]$ & {$[1,1,1,1,1]$} & $74.00 \%$ & {$[1,1,1,1,1]$} & $74.00 \%$ \\
\hline$[1,1,1,0.91,0]$ & {$[1,1,1,1,1]$} & $78.20 \%$ & {$[1,1,1,1,1]$} & $78.20 \%$ \\
\hline$[1,1,1,0.452,0]$ & {$[1,1,1,1,1]$} & $69.05 \%$ & {$[1,1,1,1,1]$} & $69.05 \%$ \\
\hline$[0,0,0,0.3,1]$ & {$[1,1,1,1,1]$} & $26.00 \%$ & {$[1,1,1,1,1]$} & $26.00 \%$ \\
\hline$R P C F_{F M T-C R I-I}$ & \multicolumn{2}{|c|}{$61.81 \%$} & \multicolumn{2}{|c|}{$61.81 \%$} \\
\hline FMP-CRI & \multicolumn{4}{|c|}{ FMP-CRI-Conclusion $B^{*}(y)$ and Reductive Property } \\
\hline Premise $A^{*}(x)$ & \multicolumn{2}{|c|}{$\begin{array}{l}\text { FMP- CRI - }{ }_{0} \\
\end{array}$} & \multicolumn{2}{|c|}{ FMP- CRI -Gougen } \\
\hline$[1,0.3,0,0,0]$ & {$[0,0,0,0.3,1]$} & $100 \%$ & {$[0,0,0,0.3,1]$} & $100 \%$ \\
\hline$[1,0.09,0,0,0]$ & {$[0,0,0,0.3,1]$} & $95.80 \%$ & {$[0,0,0,0.3,1]$} & $95.80 \%$ \\
\hline$[1,0.548,0,0,0]$ & {$[0.548,0.548,0.548,0.548,1]$} & $67.14 \%$ & {$[0,0,0,0.548,1]$} & $100 \%$ \\
\hline$[0,0.7,1,1,1]$ & {$[1,1,1,1,1]$} & $74.00 \%$ & {$[1,1,1,1,1]$} & $74.00 \%$ \\
\hline$R P C F_{F M P-C R I-I}$ & \multicolumn{2}{|c|}{$84.23 \%$} & \multicolumn{2}{|c|}{$92.45 \%$} \\
\hline FMT-CRI & \multicolumn{4}{|c|}{ FMT-CRI-Conclusion $A^{*}(x)$ and Reductive Property } \\
\hline Premise $B^{*}(y)$ & \multicolumn{2}{|c|}{ FMT-CRI-R ${ }_{0}$} & \multicolumn{2}{|c|}{ FMT-CRI-Gougen } \\
\hline$[1,1,1,0.7,0]$ & {$[1,1,1,1,1]$} & $74.00 \%$ & {$[1,1,1,1,1]$} & $74.00 \%$ \\
\hline$[1,1,1,0.91,0]$ & {$[1,1,1,1,1]$} & $78.20 \%$ & {$[1,1,1,1,1]$} & $78.20 \%$ \\
\hline$[1,1,1,0.452,0]$ & {$[1,1,1,1,1]$} & $69.05 \%$ & {$[1,1,1,1,1]$} & $69.05 \%$ \\
\hline$[0,0,0,0.3,1]$ & {$[1,1,1,1,1]$} & $26.00 \%$ & {$[1,1,1,1,1]$} & $26.00 \%$ \\
\hline$R P C F_{F M T-C R l-I}$ & \multicolumn{2}{|l|}{$61.81 \%$} & \multicolumn{2}{|c|}{$61.81 \%$} \\
\hline
\end{tabular}

In equation (38) and (39), $\rightarrow$ is implication by equation (5)-(8), $\otimes$ is its corresponding t-norm, and $\underset{x \in X}{\vee}, \underset{y \in Y}{\wedge}$ are maximum operator and minimum operator with respect to $x \in X, y \in Y$, respectively.

FMP-TIP and FMT-TIP Reductive property in Class 1 is shown in Table 3.

Table 3 FMP-TIP and FMT-TIP

Reductive property in Class 1

\begin{tabular}{l|c}
\hline FMP-TIP-Łukasiewicz & $88.73 \%$ \\
\hline FMP-TIP-Göde 1 & $92.45 \%$ \\
\hline FMP-TIP-R 0 & $84.23 \%$ \\
\hline FMP-TIP-Gougen & $92.45 \%$ \\
\hline FMT-TIP-Łukasiewicz & $44.69 \%$ \\
\hline FMT-TIP-Gödel & $44.69 \%$ \\
\hline FMT-TIP-R & $44.69 \%$ \\
\hline FMT-TIP-Gougen & $44.69 \%$ \\
\hline
\end{tabular}

From the experimental result we know that the reductive property of FMT-TIP by Gödel and Gougen are more than Lukasiewicz and $\mathrm{R}_{0}$, the reductive property of FMT-TIP is equal to all.

Next, we check the reductive property of Approximate Analogical Reasoning Schema (AARS). The AARS modifies the consequent based on the similarity (closeness) between the given premise $A^{*}$ and the antecedent $A$. In [22], distance measures (DM) for FMP (resp. FMT) is as follows.

$$
D M=D_{2}\left(A^{*}, A\right)=\left[\sum_{i=1}^{n}\left[\mu_{A^{*}}\left(x_{i}\right)-\mu_{A}\left(x_{i}\right)\right]^{2} / n\right]^{1 / 2}
$$

$$
D M=D_{2}\left(B^{*}, B\right)=\left[\sum_{i=1}^{n}\left[\mu_{B^{*}}\left(y_{i}\right)-\mu_{B}\left(y_{i}\right)\right]^{2} / n\right]^{1 / 2}
$$

The similarity by DM is then defined as follows.

$$
S_{\text {AARS }}=(1+D M)^{-1}
$$

If the rule is fired, then the consequent is modified by a modification function which could appear in one of the two forms for FMP and FMT i.e. more or less form and, fuzzy membership value reduction form, for short, reduction form, according to [22], respectively.

(FMP-AARS-more or less form)

$$
B^{*}=\min \left\{1, B / S_{\text {AARS }}\right\}
$$

(FMT-AARS-more or less form)

$$
A^{*}=\min \left\{1, A / S_{\text {AARS }}\right\}
$$

(FMP-AARS-reduction form)

$$
B^{*}=B \times S_{A A R S}
$$

(FMT-AARS-reduction form)

$$
A^{*}=A \times S_{\text {AARS }}
$$

In equation (43)-(46) $S_{\text {AARS }}$ is similarity by equation (42), min is minimum, and $x$ is algebraic product, respectively.

The reductive property of FMP-AARS and FMT-AARS shown in Table 4 are less than FMP-CRI and FMT-CRI with respect to [2], respectively.

Table 4 FMP-AARS and FMT-AARS in Class 1

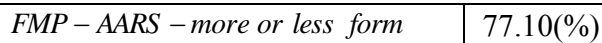




\begin{tabular}{l|l}
\hline FMP - AARS - reduction form & $76.43(\%)$ \\
\hline FMT - AARS - more or less form & $37.14 \%$ \\
\hline FMT - AARS - reduction form & $39.20 \%$ \\
\hline
\end{tabular}

Next, FMP-DM and FMT-DM reductive properties are shown in Table 5. When compared with Table 2, FMP-DM and FMT-DM reductive property are more than FMP-CRI and FMT-CRI, respectively.

\begin{tabular}{l|c}
\multicolumn{2}{c}{ Table 5 FMP-DM and FMT-DM in Class 1 } \\
\hline$F M P-D M-p(+1,0,-1)$ form & $88.06(\%)$ \\
\hline$F M P-D M-p(+1,-1)$ form & $87.64(\%)$ \\
\hline$F M T-D M-p(+1,0,-1)$ form & $88.06(\%)$ \\
\hline$F M T-D M-p(+1,-1)$ form & $92.98(\%)$ \\
\hline
\end{tabular}

Next, 13 methods are compared for FMP and FMT. (Table 6) From Table 6, in this paper, the reductive properties about TIP, CRI, and AARS are improved by our proposed DMM. From the experiment results, the reductive property ranking of the fuzzy reasoning methods in Class 1 are as follows; DMM, CRI, TIP, and AARS, respectively. Next, we compare and analyze about CRI, TIP, AARS and proposed method for Class 2.

Table 6 Comparisons of CRI, TIP, AARS DMM in Class 1

\begin{tabular}{|c|c|c|}
\hline \multicolumn{2}{|c|}{ Fuzzy Reasoning Method } & $R P C F_{F R}$ \\
\hline \multirow{2}{*}{$\begin{array}{c}\text { proposed } \\
\text { DMM }\end{array}$} & $P(+1,0,-1)$ form & $88.060 \%$ \\
\hline & $P(+1,-1)$ form & $90.310 \%$ \\
\hline \multirow{5}{*}{ CRI } & Gödel; G & $77.131 \%$ \\
\hline & Gougen; Go & $77.131 \%$ \\
\hline & Łukasiewicz; L & $75.273 \%$ \\
\hline & $\mathrm{R}_{0}$ & $73.023 \%$ \\
\hline & Zadeh; Rz & $70.098 \%$ \\
\hline \multirow{4}{*}{ TIP } & Gödel; G & $68.570 \%$ \\
\hline & Gougen; Go & $68.570 \%$ \\
\hline & Łukasiewicz & $66.711 \%$ \\
\hline & $\mathrm{R}_{0}$ & $64.461 \%$ \\
\hline \multirow{2}{*}{ AARS } & reduction form & $57.818 \%$ \\
\hline & more or less form & $57.121 \%$ \\
\hline
\end{tabular}

The reductive properties of four fuzzy reasoning methods for Class 2 are shown in Table 7.

Table 7 Comparisons of CRI, TIP, AARS DMM in Class 2

\begin{tabular}{c|l|c}
\hline \multicolumn{2}{l|}{ Fuzzy Reasoning Method } & $R P C F_{F R}$ \\
\hline \multirow{4}{*}{ CRI } & Rz & $77.83 \%$ \\
\cline { 2 - 3 } & Łukasiewicz & $77.83 \%$ \\
\cline { 2 - 3 } & Gödel & $86.38 \%$ \\
\cline { 2 - 3 } & $\mathrm{R}_{0}$ & $77.83 \%$ \\
\cline { 2 - 3 } & Gougen & $86.38 \%$ \\
\hline \multirow{4}{*}{ TIP } & Łukasiewicz & $62.01 \%$ \\
\cline { 2 - 3 } & Gödel & $62.08 \%$ \\
\cline { 2 - 3 } & Ro & $57.95 \%$ \\
\cline { 2 - 3 } & Gougen & $62.08 \%$ \\
\hline \multirow{2}{*}{ AARS } & more or less & $56.59 \%$ \\
\cline { 2 - 3 } & Reduction & $57.25 \%$ \\
\hline \multirow{2}{*}{ Proposed } & $P(+1,0,-1)$ form & $95.02 \%$ \\
\cline { 2 - 3 } DMM & $P(+1,-1)$ form & $91.55 \%$ \\
\hline
\end{tabular}

In Table 7, the given premises of fuzzy rule (1) are $A^{*}=$ s.t. $A=[1,0.2,0,0,0], \quad B^{*}=$ s.t. $B=[0,0,0,0.2,1]$. From Table 7 , we can see that, the best is our proposed method, next best CRI, TIP, and the lowest AARS in Class 2, this result is similar as in Class 1. The reductive properties of the 4 fuzzy reasoning methods are comprehensively shown in Table 8.

Table 8 The comprehensive reductive properties of the 4 fuzzy reasoning methods for Class 1 and 2

\begin{tabular}{c|c|c|c|c}
\hline Method & DMM & CRI & TIP & AARS \\
\hline FMP (\%) & 92.430 & 87.719 & 92.458 & 86.700 \\
\hline FMT (\%) & 92.428 & 69.555 & 35.195 & 27.663 \\
\hline Average (\%) & 92.429 & 78.637 & 63.827 & 57.182 \\
\hline
\end{tabular}

Through the experiments we have obtained that proposed FMP-DM and FMT-DM methods are in accordance with human thinking.

\section{Comparison with Respect to Fuzzy Control}

In this section we compare with [17]'s fuzzy reasoning method based on fuzzy relation and our proposed method based on distance measure with respect to fuzzy control. Let us consider [16]'s method and [32]'s CRI by the equation (1), (2). According to [16] fuzzy reasoning style is based on the idea converting the fuzzy conditional sentence of $《$ if $x$ is $A$ then $y$ is $B 》$ to the fuzzy relation for FMP and FMT. That is,

$$
\langle\langle x \text { is } A\rangle \rightarrow\langle\langle y \text { is } B\rangle=\sqrt{ }(x, y) \text { is } R\rangle
$$

In fuzzy control, 2 antecedents are usually used as follows.

$$
\left\langle\left\langle x_{1} \text { is } A_{1}\right\rangle\right\rangle \text { and }\left\langle x_{2} \text { is } A_{2}\right\rangle \rightarrow\langle\langle y \text { is } B\rangle
$$

Equation (48) is divided as equation (49).

$$
\begin{aligned}
\left\langle x_{1} \text { is } A_{1}\right\rangle & \left.\rightarrow\langle y \text { is } B\rangle=\sqrt{ }\left(x_{1}, y\right) \text { is } R\right\rangle \\
\left\langle x_{2} \text { is } A_{2}\right\rangle & \left.\rightarrow\langle y y \text { is } B\rangle=\sqrt{ }\left(x_{2}, y\right) \text { is } R\right\rangle
\end{aligned}
$$

By expressing like this, we can consider $(x, y)$ to the names of objects, $R$ is the predicate, and this is a Theorem. Denoting fuzzy rule if $x$ is $A$ then $y$ is $B\rangle$ as $A \rightarrow B$, then fuzzy relation $A \rightarrow B$ is defined as fuzzy implication $\mu_{A \rightarrow B}(u, v)=\mu_{A}(u) \rightarrow \mu_{B}(v)$. At this time, $\mathrm{R}$ may be changed according to what implication $A \rightarrow B$ is used. They are as follows.

$$
\begin{aligned}
R_{\mathrm{m}} & =(A \times B) \bigcup((1-A) \times V)= \\
& =\int_{U \times V}\left[\mu_{A}(u) \wedge \mu_{B}(v) \wedge\left(1-\mu_{A}(u)\right) /(u, v)\right. \\
R_{a} & =\neg A \oplus B=\int_{U \times V}\left[1 \wedge\left(1-\mu_{A}(u)+\mu_{B}(v)\right)\right] /(u, v) \\
R_{\mathrm{c}} & =A \times B=\int_{U \times V} \mu_{A}(u) \wedge \mu_{B}(v) /(u, v) \\
R_{p} & =A \times B=\int_{U \times V} \mu_{A}(u) \mu_{B}(v) /(u, v)
\end{aligned}
$$

Using fuzzy relation $R_{c}, R_{m}, R_{a}$ instead of $A \rightarrow B$, conclusion $B^{\prime}$ is obtained by FMP-CRI. In the same way, conclusion $A^{*}$ is obtained by 
FMT-CRI. There are $R_{g}, R_{s}$ methods beside fuzzy relation $R_{c}, R_{m}, R_{a}$. In [16] they are as follows.

$$
\begin{aligned}
& R_{s}=A \times V \longrightarrow U \times B=\int_{U \times V} \mu_{A}(u) \longrightarrow \mu_{B}(v)(u, v) \\
& \mu_{A}(u) \longrightarrow \mu_{B}(v)= \begin{cases}1, & \text { if } \mu_{A}(u) \leq \mu_{B}(v) \\
0, & \text { if } \mu_{A}(u)>\mu_{B}(v)\end{cases} \\
& R_{g}=A \times V \longrightarrow \mathrm{g} U \times B=\iint_{U \times V}(u) \underset{\mathrm{g}}{\longrightarrow} \mu_{B}(v)(u, v) \\
& \mu_{A}(u) \underset{\mathrm{g}}{\longrightarrow} \mu_{B}(v)=\left\{\begin{array}{lll}
1, & \text { if } \mu_{A}(u) \leq \mu_{B}(v) \\
\mu_{B}(v), & \text { if } & \mu_{A}(u)>\mu_{B}(v)
\end{array}\right.
\end{aligned}
$$

By combining fuzzy relation $R_{s}$ and $R_{g}$, four fuzzy relations are obtained. And by introducing the accommodation in multi-value logic, several fuzzy relations are obtained. The several fuzzy reasoning results calculated for FMP and FMT presented in [17] are shown in Table 9 and 10 , respectively. By the same way in different cases fuzzy reasoning results and reductive property criterion functions are calculated according to proposed DMM. Total calculated results are shown in Table 11 for FMP and FMT. Comparison of $[13,17]$ 's and DMM is shown in Table 12.

Table 9 FMP reasoning results by different fuzzy relation ([See 16,17])

\begin{tabular}{l|c|c|c|c}
\hline \multicolumn{1}{c|}{ Fuzzy reasoning } & $A$ & very $A$ & more or less $A$ & not $A$ \\
\hline$R_{m}[32]$ & $\frac{1}{2} \vee \mu_{B}$ & $\frac{1}{2}(\sqrt{5}-1) \vee \mu_{B}$ & $\frac{1}{2}(3-\sqrt{5}) \vee \mu_{B}$ & 1 \\
\hline$R_{a}[32]$ & $\frac{1}{2}\left(1+\mu_{B}\right)$ & $\frac{1}{2}\left(3+2 \mu_{B}-\sqrt{5-4 \mu_{B}}\right)$ & $\frac{1}{2} \sqrt{5+4 \mu_{B}-1}$ & 1 \\
\hline$R_{c}[13]$ & $\mu_{B}$ & $\mu_{B}$ & $\mu_{B}$ & $\frac{1}{2} \wedge \mu_{B}$ \\
\hline$R_{s}, R_{g}[17]$ & $\mu_{B}$ & $\mu_{B}^{2}$ & $\mu_{B}^{0.5}$ & 1 \\
\hline$R_{s g}, R_{s s} R_{g s}, R_{g g}[17]$ & $\mu_{B}$ & $\mu_{B}^{2}$ & $\mu_{B}^{0.5}$ & $1-\mu_{B}$ \\
\hline
\end{tabular}

\begin{tabular}{|c|c|c|c|}
\hline \multicolumn{2}{|c|}{ Antecedent; $A=[0,0.25,0.5,0.75,1]$} & \multicolumn{2}{|c|}{ Consequent; $B=[0,0.25,0.5,0.75,1]$} \\
\hline \multicolumn{2}{|c|}{ The given premise; $A^{*}(x)$} & Conclusion; $B^{*}(y)=$ ? & RPCF \\
\hline$A=[$ large $]$ & $A^{*}=[0,0.250,0.500,0.750,1]$ & $B^{*}=[0,0.250,0.500,0.750,1]$ & $100 \%$ \\
\hline very $A=[\text { large }]^{2}$ & $A^{*}=[0,0.063,0.250,0.563,1]$ & $B^{*}=[0,0.087,0.337,0.587,1]$ & $97.28 \%$ \\
\hline more or less $A=[\text { large }]^{\frac{1}{2}}$ & $A^{*}=[0,0.500,0.707,0.866,1]$ & $B^{*}=[0,0.404,0.654,0.904,1]$ & $96.26 \%$ \\
\hline not $A=1-[$ large $]$ & $A^{*}=[1,0.750,0.500,0.250,0]$ & $B^{*}=[0.727,1,0.500,0,0.273]$ & $79.06 \%$ \\
\hline \multicolumn{3}{|c|}{ RPCF-FMP-DM-average } & $93.15 \%$ \\
\hline \multicolumn{2}{|c|}{ Fuzzy Rule Antecedent; $1-B=[1,0.750,0.500,0.250,0]$} & \multicolumn{2}{|c|}{ Consequent; $1-A=[1,0.75,0.5,0.25,0]$} \\
\hline \multicolumn{2}{|c|}{ The Given Premise; $B^{*}(y)$} & Conclusion; $A^{*}(x)=?$ & RPCF \\
\hline not $B=1-[$ large $]$ & $B^{*}=[1,0.750,0.500,0.250,0]$ & $A^{*}=[1,0.750,0.500,0.250,0]$ & $100 \%$ \\
\hline not very $B=1-[\text { large }]^{2}$ & $B^{*}=[1,0.938,0.750,0.438,0]$ & $A^{*}=[1,0.913,0.663,0.413,0]$ & $97.28 \%$ \\
\hline not more or less $B=1-[\text { large }]^{\frac{1}{2}}$ & $B^{*}=[1,0.500,0.293,0.134,0]$ & $A^{*}=[1,0.596,0.346,0.096,0]$ & $96.26 \%$ \\
\hline$B=[$ large $]$ & $B^{*}=[0,0.250,0.500,0.750,1]$ & $A^{*}=[0.273,0,0.500,1,0.727]$ & $79.06 \%$ \\
\hline
\end{tabular}

Table 10 FMT reasoning results by different fuzzy relation ([See 16,17])

\begin{tabular}{c|c|c|c|c}
\hline Fuzzy reasoning & not $B$ & not very $B$ & not more or less $B$ & $B$ \\
\hline$R_{m}[32]$ & $0.5 \vee \overline{\mu_{A}}$ & $\left(\overline{\mu_{A}} \vee \frac{1}{2}(\sqrt{5}-1)\right) \vee \mu_{A}$ & $\frac{1}{2}(3-\sqrt{5}) \vee\left(1-\mu_{A}\right)$ & $\mu_{A} \vee \bar{\mu}_{A}$ \\
\hline$R_{a}[32]$ & $1-\frac{1}{2} \mu_{A}$ & $\frac{1}{2}\left(1-2 \mu_{A}+\sqrt{1+4 \mu_{A}}\right)$ & $\frac{1}{2}\left(3-\sqrt{1+4 \mu_{A}}\right)$ & 1 \\
\hline$R_{c}[13]$ & $0.5 \wedge \mu_{A}$ & $\frac{1}{2}(\sqrt{5}-1) \wedge \mu_{A}$ & $\frac{1}{2}(3-\sqrt{5}) \wedge \mu_{A}$ & $\mu_{A}$ \\
\hline$R_{s}[17]$ & $1-\mu_{A}$ & $1-\mu_{A}^{2}$ & $1-\mu_{A}^{0.5}$ & 1 \\
\hline$R_{g}[17]$ & $0.5 \vee \overline{\mu_{A}}$ & $\frac{1}{2}(\sqrt{5}-1) \vee\left(1-\mu_{A}^{2}\right)$ & $\frac{1}{2}(3-\sqrt{5}) \vee\left(1-\mu_{A}^{0.5}\right)$ & 1 \\
\hline$R_{s g}[17]$ & $1-\mu_{A}$ & $1-\mu_{A}^{2}$ & $1-\mu_{A}^{0.5}$ & $0.5 \vee \mu_{A}$ \\
\hline$R_{g g}[17]$ & $0.5 \vee \overline{\mu_{A}}$ & $\frac{1}{2}(\sqrt{5}-1) \vee\left(1-\mu_{A}^{2}\right)$ & $\frac{1}{2}(3-\sqrt{5}) \vee\left(1-\mu_{A}^{0.5}\right)$ & $0.5 \vee \mu_{A}$ \\
\hline$R_{g s}[17]$ & $0.5 \vee \overline{\mu_{A}}$ & $\frac{1}{2}(\sqrt{5}-1) \vee\left(1-\mu_{A}^{2}\right)$ & $\frac{1}{2}(\sqrt{5}-1) \vee\left(1-\mu_{A}^{2}\right)$ & $\mu_{A}$ \\
\hline$R_{s s}[17]$ & $1-\mu_{A}$ & $1-\mu_{A}^{2}$ & $1-\mu_{A}^{0.5}$ & $\mu_{A}$ \\
\hline
\end{tabular}

Table 11 Proposed FMP-DM and FMT-DM Reductive Property for [17]'s problem 
Table 12 Comparison of [13,17]'s and proposed method

\begin{tabular}{c|c|c|c|c|c|c}
\hline No & Fuzzy Reasoning Method & $\begin{array}{c}\text { Reductive Property } \\
\text { For FMP }\end{array}$ & $\begin{array}{c}\text { Reductive Property } \\
\text { For FMT }\end{array}$ & $\begin{array}{c}\text { Reductive } \\
\text { Property }\end{array}$ & $\begin{array}{c}\text { Fuzzy Control } \\
\text { Capability }\end{array}$ \\
\hline 1 & Mizumoto [17] & $R_{s s}$ & $100 \%$ & $100 \%$ & $100 \%$ & No \\
\hline 2 & Proposed DMM & DMM & $93.15 \%$ & $93.15 \%$ & $93.15 \%$ & Yes \\
\hline 3 & Mizumoto [17] & $R_{s g}$ & $100 \%$ & $75 \%$ & $87.5 \%$ & No \\
\hline 4 & Mizumoto [17] & $R_{s}$ & $75 \%$ & $75 \%$ & $75 \%$ & No \\
\hline 5 & Mizumoto [17] & $R_{g g}$ & $75 \%$ & $0 \%$ & $37.5 \%$ & No \\
\hline 6 & Mizumoto [17] & $R_{g s}$ & $75 \%$ & $0 \%$ & $37.5 \%$ & No \\
\hline 7 & Mizumoto [17] & $R_{g}$ & $50 \%$ & $0 \%$ & $25 \%$ & No \\
\hline 8 & Mamdani [13] & $R_{c}$ & $25 \%$ & $0 \%$ & $12.5 \%$ & Yes \\
\hline
\end{tabular}

Next let us about the fuzzy control based on different fuzzy reasoning methods. As shown in [33], the mathematical model of the control object is as follows.

$$
G(s)=[1 /(1+T S)] \cdot \exp (-\tau), \quad T=20(s), \tau=2(s)
$$

where target value is $r=40$, sampling time $t=1$ (s), error $e=r-y(t)$, change of error $\Delta e=y(t-1)-y(t)$. The increment $\Delta u$ of fuzzy control obtained by fuzzy reasoning is calculated as follows.

$$
u(k)=u(k-1)+\rho \cdot \Delta u(k)
$$

where, parameter $\rho$ is amplification coefficient, $k$ discrete time. Analysis for the reductive property of 14 fuzzy reasoning methods from [17] and proposed DMM can be summarized as follows. From Table 11 the reductive property of the fuzzy reasoning method $R_{s s}(100 \%)$ is more than proposed DMM (93.15\%). However the reductive property of $R_{s s}, R_{s}, R_{g g}, R_{g s}, R_{g}$, and $R_{c} \quad(87.5 \%, 75 \%, 37.5 \%, 37.5 \%, 25 \%$, and $12.5 \%)$ are less than proposed DMM, respectively. And since the fuzzy reasoning methods $R_{m}, R_{a}, R_{\#}$, $R_{\Delta}, R_{\mathrm{v}}$, and $R_{*}$ do not satisfy the reductive property, which cannot be applied to the practical problems, for example fuzzy control. The reductive properties of fuzzy reasoning methods for $R_{s s}, R_{s s}, R_{s}, R_{c}$, and proposed DMM are shown in Fig. 1 .

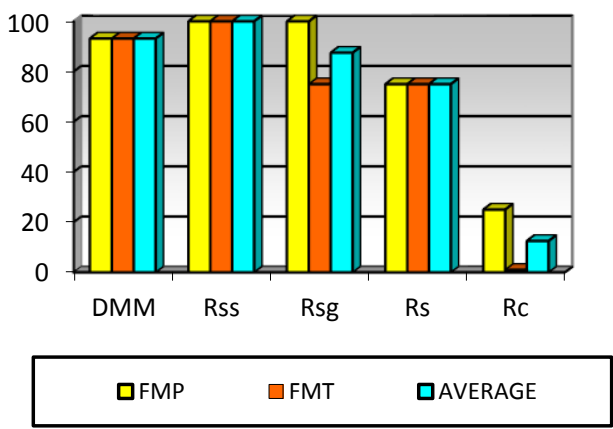

Fig. 1 The reductive properties of fuzzy reasoning methods for $R_{s s}, R_{s g}, R_{s}, R_{c}$ and DMM

According to the experimental result, proposed DMM, $R_{c}, R_{m}, R_{a}$, and so on, can be applied to fuzzy control. Especially the fuzzy reasoning method $R_{c}$ presented by E. H. Mamdani in [19] was widely used not only fuzzy control but also pattern recognition, expert system, modeling, predication, system analysis, diagnosis, retrieval system, learning system, and so on. As mentioned in [13-16], the fuzzy relation based reasoning methods $R_{s s}, R_{s g}, R_{s}, R_{g g}, R_{g s}$, and $R_{g}$ cannot be applied to fuzzy control.

Those of reasons can be described as follows.

Theorem 5.1. Fuzzy reasoning methods based on the fuzzy relation $R_{s}$ and $R_{g}$ do not satisfy the convergence of the fuzzy control.

\section{Proof.}

Let $u_{0} \in U$ be crisp input information, $\mu_{A^{\prime}}\left(u_{0}\right)$ membership function fuzzificated by $u_{0}$. For every crisp information $u_{0}$, the fuzzy reasoning result by $R_{s}$ and $R_{g}$ are always obtained as 1 or 0 for $R_{s}$, and 1 or $\mu_{B}(v)$ for $R_{g}$, that is, according to CRI [32], conclusions $\mu_{B^{*}}(v)$ of fuzzy reasoning method $R_{s}$ and $R_{g}$ are calculated as follows, respectively.

$$
\begin{aligned}
& \mu_{B^{*}}(v)=\underset{u}{\vee}\left\{\mu_{A^{\prime}}\left(u_{0}\right) \wedge\left[\mu_{A}(u) \underset{s}{\rightarrow} \mu_{B}(v)\right]\right\}= \\
& =\underset{u}{\vee}\left\{\mu_{A^{\prime}}\left(u_{0}\right) \wedge \mu_{A}(u)\right\} \underset{s}{\rightarrow} \mu_{B}(v)= \\
& =h \rightarrow \underset{s}{\rightarrow} \mu_{B}(v)= \begin{cases}1, & h \leq \mu_{B}(v) \\
0, & h>\mu_{B}(v)\end{cases} \\
& \mu_{B^{*}}(v)=\underset{u}{\vee}\left\{\mu_{A^{\prime}}\left(u_{0}\right) \wedge\left[\mu_{A}(u) \underset{g}{\rightarrow} \mu_{B}(v)\right]\right\}= \\
& =\underset{u}{\vee}\left\{\mu_{A^{\prime}}\left(u_{0}\right) \wedge \mu_{A}(u)\right\} \underset{g}{\rightarrow} \mu_{B}(v)=h \rightarrow \mu_{B}(v)= \\
& = \begin{cases}1, & h \leq \mu_{B}(v) \\
\mu_{B}(v), & h>\mu_{B}(v)\end{cases}
\end{aligned}
$$


, where $h=\underset{u}{\vee}\left\{\mu_{A^{\prime}}\left(u_{0}\right) \wedge \mu_{A}(u)\right\}$ is a degree of matching of the fuzzy rule. From the equation (74) and (75) we can know that when input information $\mu_{A^{\prime}}\left(u_{0}\right)$ is changed according to $u_{0}$ then $\mu_{B^{*}}(v)$ is not changed and fixed as crisp value 1 and 0 , and fuzzy set $\mu_{B}(v)$, therefore the convergence of the fuzzy control cannot be guaranteed.

As mentioned in $[16,17]$ these are logical contradiction between the reductive property (i.e., human thinking) and the practical problem (e.g. fuzzy control).

Theorem 5.2. Fuzzy reasoning methods based on the fuzzy relation $R_{s s}, R_{s g}, R_{s}, R_{g g}, R_{g s}$, and $R_{g}$ do not satisfy the convergence of the fuzzy control.

\section{Proof.}

From Theorem 5.1 we can easily know the convergence of the fuzzy control. When the fuzzy relation $R_{s s}, R_{s g}, R_{s}, R_{g g}, R_{g s}$, and $R_{g}$ are applied to fuzzy control, for different input information different reasoning results are not obtained, but same ones are calculated, respectively. That is, those have not their convergence. This is illustratively proofed by the extension of equation (74) and (75).

Theorem 5.3. Fuzzy reasoning methods based on the fuzzy relation $R_{c}, R_{m}, R_{p}$ and $R_{a}$ do satisfy the convergence of the fuzzy control.

\section{Proof.}

For different input information, different reasoning results are obtained by $R_{c}, R_{m}, R_{p}$ and $R_{a}$. For simplicity let us consider only the fuzzy relation $R_{c}$. For crisp information $u_{0} \in U$, let $h_{i}=\underset{u}{\vee}\left\{\mu_{A^{\prime}}\left(u_{0}\right) \wedge \mu_{A_{i}}(u)\right\}$ be degree of matching of the ith rule, and $i=1,2, \cdots, n$ number of rules, then the individual fuzzy reasoning results by $R_{c}$ are as follows.

$$
\begin{aligned}
& \mu_{B_{i}^{*}}(v)=\underset{u}{\vee}\left\{\mu_{A^{\prime}}\left(u_{0}\right) \wedge\left[\underset{A_{A_{i}}}{ }(u) \underset{c}{\rightarrow} \mu_{B_{i}}(v)\right]\right\}= \\
& =\underset{u}{\vee}\left\{\mu_{A^{\prime}}\left(u_{0}\right) \wedge \mu_{A_{i}}(u)\right\} \underset{c}{\rightarrow} \mu_{B_{i}}(v)=h_{i} \wedge \mu_{B_{i}}(v)
\end{aligned}
$$

The final fuzzy reasoning result $\mu_{B^{*}}(v)$ by fuzzy relation $R_{c}$ is calculated as follows.

$$
\mu_{B^{*}}(v)=\bigcup_{i=1}^{n}\left\{h_{i} \wedge \mu_{B_{i}}(v)\right\}
$$

From equation (77), we can see that when degree of matching $h_{i}$ is changed then $\mu_{B^{*}}(v)$ is also changed according to $h_{i}$, therefore the fuzzy reasoning by Mamdani's $R_{c}$ [13] does satisfy the convergence of the fuzzy control. $\square$

Theorem 5.4. Proposed method DMM does satisfy the convergence of the fuzzy control and has not information loss with respect to [2, 33].

\section{Proof.}

For different input information, different reasoning results are obtained by proposed method DMM. From Definition 3.10, for every crisp input information $u_{0} \in U$, Euclidian distance measures $D M\left(A\left(u_{0}\right), A_{i}(u)\right)$ between the given premise and ith rule are not equal to. That is,

$$
\begin{aligned}
& D M\left(\mu_{A}\left(u_{0}\right), \mu_{A_{1}}(u)\right) \neq D M\left(\mu_{A}\left(u_{0}\right), \mu_{A_{2}}(u)\right) \neq \cdots \\
& \neq D M\left(\mu_{A}\left(u_{0}\right), \mu_{A_{i}}(u)\right) \neq \cdots \neq D M\left(\mu_{A}\left(u_{0}\right), \mu_{A_{n}}(u)\right)
\end{aligned}
$$

From the proposed method, $v_{0} \in V$ satisfying following equation must be obtained.

$$
D M\left(\mu_{B}\left(v_{0}\right), \mu_{B_{i}}(v)\right)=D M\left(\mu_{A}\left(u_{0}\right), \mu_{A}(u)\right)
$$

Our aim is to find $v_{0}$ satisfying the equation (73). Then Euclidian distance measures $D M\left(\mu_{B}\left(v_{0}\right), \mu_{B}(v)\right)$ between the fuzzy reasoning conclusion and the consequent of $i$ th fuzzy rule are obtained as follows.

$$
\begin{aligned}
& D M\left(\mu_{B}\left(v_{0}\right), \mu_{B_{i}}(v)\right) \neq D M\left(\mu_{B}\left(v_{0}\right), \mu_{B_{2}}(v)\right) \neq \cdots \\
& \neq D M\left(\mu_{B}\left(v_{0}\right), \mu_{B_{i}}(v)\right) \neq \cdots \neq D M\left(\mu_{B}\left(v_{0}\right), \mu_{B_{n}}(v)\right)
\end{aligned}
$$

Where $v_{0} \in V$ is defuzzificated value of the fuzzy reasoning result. Thereby the fuzzy reasoning results are different. In other words for small input information, corresponding small reasoning results are obtained, whereas, for big input information, corresponding big reasoning results are obtained, which means that reasoning method has in itself convergence. Our method does satisfy the reductive property and can be applied to the fuzzy control and so on.

Proposed method DMM has not information loss. Its reason is as follows. Let quasi-fuzzy reasoning result be $\widetilde{B}_{i}$, then following equation is satisfied.

$$
\mu_{\tilde{B}_{1}}(v)=\mu_{B_{1}}(v)+D M\left(\mu_{A^{\prime}}\left(u_{0}\right), \mu_{A}(u)\right) \times P_{i}
$$

Thus the fuzzy reasoning result of ith rule is calculated as follows from Definition 3.13.

$$
\mu_{B_{i}^{*}}(v)=\frac{\mu_{\widetilde{B}_{i}}(v)-\eta_{i}}{\xi_{i}-\eta_{i}}
$$

Where $\xi_{i}=\max _{1} \tilde{B}_{i}$ and $\eta_{i}=\min \widetilde{B}_{i}$ are maximum and minimum of the quasi-fuzzy reasoning result, respectively. By these operations the information losses are overcame. Since the standardization operation is used in equation (82), thereby our proposed DMM has not any information loss. Final 
crisp reasoning result $v_{0}$ mentioned in equation (79) and (80) is calculated as follows.

$$
v_{0}=\frac{\sum_{i=1}^{n} B_{i}^{*}\left(v_{i}\right) \times v_{i}}{\sum_{i=1}^{n} B_{i}^{*}\left(v_{i}\right)}
$$

Where $v_{0}, v_{i} \in V$. From equation (77) we can see that $v_{0}$ has not the information loss. Thus the proof of this Theorem 5.4 is completed. $\square$

From the theorem 5.1-5.4, we know that proposed method DMM and $R_{c}$ by Mamdani have control capacity, whereas, $R_{s g}, R_{s}, R_{g g}, R_{g s}, R_{g}$ have not it.

\section{Conclusions}

This paper shows a basic and original fuzzy reasoning method that can draw a novel study direction of the approximate inference in fuzzy systems with uncertainty. Our research results can be summarized as follows.

We first proposed reductive property criterion function for checking of the fuzzy reasoning result. And then, unlike well-known fuzzy reasoning methods based on the similarity measure, we proposed a principle of new fuzzy reasoning method based on distance measure, for short, DMM, and then presented two theorem for FMP and FMT.

The CRI, TIP and AARS use not only linear operators but also nonlinear operators, thus they have the information loss in fuzzy reasoning. Otherwise our method uses linear operators, which has not the information loss in fuzzy reasoning, and is more than CRI, TIP and AARS with respect to the reductive property. We compared 13 fuzzy reasoning methods for FMP and FMT. Consequently our proposed DMM is illustratively better than AARS, TIP, and CRI with respect to the reductive property, and in accordance with human thinking.

We discussed that fuzzy reasoning methods based on the fuzzy relation $R_{s}$ and $R_{g}$ do not satisfy the convergence of the fuzzy control, whereas, fuzzy reasoning methods based on the fuzzy relation $R_{s s}, R_{s g}, R_{s}, R_{g g}, R_{g s}$, and $R_{g}$ do not satisfy the convergence of the fuzzy control. And we fined that fuzzy reasoning methods based on the fuzzy relation $R_{c}, R_{m}, R_{p}$ and $R_{a}$ do satisfy the convergence of the fuzzy control.

Consequently we pointed out conclusion that proposed method does satisfy the convergence of the fuzzy control and has not information loss.

\section{References:}

[1] L. Bloc, P. Borowik, Many-Valued Logic, Springer -Verlag. Warsawa, 1993.

[2] J. Buckley, Y. Hayashi, Can approximate reasoning be consistent? , Fuzzy Sets and Systems, Vol. 65, 1994, pp. 13-18.

[3] S. M. Chen, A new approach to handling fuzzy decision-making problems, IEEE Trans. Syst. Man Cybern., Vol. 18, 1988, pp. 1012-1016.

[4] S. M. Chen, A weighted fuzzy reasoning algorithm for medical diagnosis, Decis. Support Syst., Vol. 11, 1994, pp. 37-43.

[5] Guannan Deng, Yanli Jiang, Fuzzy reasoning method by optimizing the similarity of truth-tables, Information Sciences, Vol. 288, 2014, pp. 290-313.

[6] D. Dubois, H. Prade, Fuzzy Sets and Systems, Academic Press, New York, 1980.

[7] HE Ying-Si, QUAN Hai-Jin, DENG Hui-Wen, An Algorithm of General Fuzzy Inference With The Reductive Property, Computer Science, Vol. 34, 2007, pp. 145-148.

[8] Y. S. He, H.J. Quan, Study on the results of triple I method, Computer Sciences, Vol. 39, 2012, pp. 248-250.

[9] J. Jantzen, Foundations of Fuzzy Control, John Wiley and Sons, Chichester, 2007.

[10] Son-Il Kwak, Un-Sok RYU, Kum-Ju KIM, and Myong-Hye JO, A Fuzzy reasoning Method based on Compensationg Operation and its Application to Fuzzy Systems, Iranian Journal of Fuzzy Systems, 16(3), pp. 17-34, 2019.

[11] M. X. Luo, and N. Yao, Triple I algorithms based on Schweizer-Sklar operators in fuzzy reasoning, International Journal of Approximate Reasoning, Vol. 54, 2013, pp. 640-652.

[12] Minxia Luo and Kaiyan Zhou, Logical foundation of the quintuple implication inference methods, International Journal of Approximate Reasoning, Vol. 101, 2018, pp. 1-9.

[13] E. H. Mamdani, Application of fuzzy logic to approximate reasoning using linguistic systems, IEEE Transactions on Computers, Vol. 26, 1977, pp. 1182-1191.

[14] M. Mizumoto, Fuzzy inference using $\max -^{\wedge}$ composition in the compositional rule of inference, In: M.M. Gupta, E. Sanchez (Eds.), Approximate Reasoning in Decision Analysis, North Holland, Amsterdam, 1982, pp. 67-76.

[15] M. Mizumoto, Fuzzy conditional inference under max-composition, Information Sciences, 
Vol. 27, 1982, pp. 183-209.

[16] M. Mizumoto, Extended fuzzy reasoning, In: M.M. Gupta, A. Kandel, W. Bandler, J.B. Kiszka (Eds.), Approximate Reasoning in Expert Systems, North Holland, Amsterdam, 1985, pp. 71-85.

[17] M. Mizumoto, Improvement of fuzzy control, $3^{\text {th }}$ Fuzzy System Symposium, 1987, pp. $153-158$

[18] M. Mizumoto, H. J. Zimmermann, Comparison of fuzzy reasoning methods, Fuzzy Sets and Systems, Vol. 8, 1982, pp. 253-283.

[19] W. Pedrycz, F. Gomide, Introduction to Fuzzy Sets, MIT Press. Cambridge, MA, 1998.

[20] S. J. Song, C. Wu: Reverse triple I method of fuzzy reasoning, Sci. China (Series F), Vol. 45, 2002, pp. 344-364.

[21] I. B. Turksen, Z. Zhong, An approximate analogical reasoning scheme based on similarity measures and interval valued fuzzy sets, Fuzzy Sets and Systems, Vol. 34, 1990, pp. 323-346.

[22] I. B. Turksen, Z. Zhong, An approximate analogical reasoning approach based on similarity measures, IEEE Trans. Syst. Man Cybern. Vol. 18, 1988, pp. 1049-1056.

[23] De-Gang Wang, Yan-Ping Meng, Hong-Xing Li, A fuzzy similarity inference method for fuzzy reasoning, Computers and Mathematics with Applications, Vol. 56, 2008, pp. 2445-2454.

[24] G. J. Wang, On the logic foundations of fuzzy modus ponens and fuzzy modus tollens, Journal Fuzzy Mathematics, Vol. 5, 1997, pp. 229.

[25] G. J. Wang, Logic on certain algebras (I), $J$. Shaanxi Normal University, vol. 25, 1997.

[26] G. J. Wang, The triple I method with total inference rules of fuzzy reasoning, Science in China, Vol. 29, 1999, pp. 43-53.

[27] G. J. Wang, Formalized theory of general fuzzy reasoning, Information Sciences, Vol. 160,
2004, pp. 251-266.

[28] D. S. Yeung, E.C.C. Tsang, A comparative study on similarity based fuzzy reasoning methods, IEEE Trans. on Systems, Man, and Cybernetics Part B: Cybernetics, Vol. 27, 1997, pp. 216-227.

[29] D. S. Yeung, E.C.C. Tsang, Improved fuzzy knowledge representation and rule evaluation using fuzzy Petri nets and degree of subsethood, Intelligence Systems, Vol. 9, 1994, pp. 1083-1100.

[30] Xue-hai Yuan, E. Stanley Lee, Comments on Notes on triple I method of fuzzy reasoning, Computers and Mathematics with Applications, Vol. 58, 2009, pp. 1604-1607.

[31] Z. H. Zhao, Y.J. Li, Reverse triple I method of fuzzy reasoning for the implication operator RL, Computers and Mathematics with Applications, Vol. 53, 2007, pp. 1020-1028.

[32] L. A. Zadeh, The concept of a linguistic variable and its applications to approximate reasoning, I, Information Sciences, Vol. 8, 1975, pp. 199-249.

[33] M. C. Zheng, Z. K. Shi, Y. Liu, Triple I method of approximate reasoning on Atanassovs intuitionistic fuzzy sets, International Journal of Approximation Reasoning,. 2014, http://dx.doi.org/10.1016/j.ijar. 2014. 01.001

[34] B. Zhou, Genqi Xu, and Sanjiang Li, The Quintuple Implication Principle of fuzzy reasoning, Information Sciences, Vol. 297, 2015, pp. 202-215.

\section{Creative Commons Attribution License 4.0 (Attribution 4.0 International, CC BY 4.0)}

This article is published under the terms of the Creative Commons Attribution License 4.0 https://creativecommons.org/licenses/by/4.0/deed.en US 